\title{
Edificación residencial, crowding out y política de viviendas
}

\author{
Montserrat Díaz \\ Mar Llorente \\ Universidad de Oviedo \\ Paloma Taltavull \\ Universidad de Alicante
}

\section{Resumen}

El artículo desarrolla un análisis para la identificación de efectos crowding entre las viviendas iniciadas privadas y públicas en España y en 8 provincias, identificando efectos de expulsión (crowding out) o de empuje (crowding in) de la construcción privada ante acciones de incentivo a la edificación pública. La metodología utiliza el entorno de vectores autorregresivos estimando las relaciones de cointegración entre ambos ciclos. Los resultados identifican efectos de crowding out en Cáceres y Madrid, efectos similares pero en dirección contraria, desde la edificación privada impulsando la pública en Vizcaya; no se identifican efectos estadísticamente significativos en Guadalajara y Zaragoza y un efecto crowding in en Alicante en las dos direcciones.

Palabras clave: edificación pública y privada, crowding out, España, política de vivienda.

Clasificación JEL: R31, R38.

\begin{abstract}
This paper analyses the existence of crowding effects between the public and private housing cycles in eight selected Spanish provinces. It identifies the crowding out and crowding in effects in private house development cycle when incentives to public unit construction appears. Using Autorregressive Vector and cointegration methodologies, the exercise allows to estimate the influence and causality in two directions, that is, how any impulse in private hose-building affect to public house starts and how public incentives constructing dweelings can push the private construction. The paper finds the existence of crowding out effects between public towards private house-building in Cáceres and Madrid; similar effects but in the inverse direction have been found in Vizcaya where the private building is crowding in public house-building. There is no crowding effect, in any direction, identified in Guadalajara and Zaragoza and there is a strong two-directions crowding-in effect reinforcing house-building in Alicante.
\end{abstract}

Keywords: public house-building, private construction, starts, crowding out, Spain, housing policy.

JEL classification: R31, R38. 


\section{Introducción}

Desde mediados de la década de los noventa hasta el inicio de la última recesión, la mayoría de las economías desarrolladas se caracterizaron por un notable incremento de la inversión residencial, acompañada de significativos aumentos en el nivel de precios de la vivienda, tendencia que se invierte dando lugar a un ajuste sustancial de los precios y de las cantidades a partir del año 2008.

El fuerte dinamismo de la inversión residencial en España durante el periodo de expansión y la intensa corrección posterior, constituyen aspectos claves de su ciclo inmobiliario. Después de la crisis de 1989-1992 la recuperación del sector inmobiliario es temprana, en 1994 comienza la fase de crecimiento que alcanza la cota máxima en el año 2006 con más de 700.000 unidades de vivienda iniciadas. Entre 1998 y 2007, los precios de la vivienda en España se triplicaron en términos nominales y el número medio de las hipotecas concedidas superó el millón anual, el crecimiento del sector de la construcción explicó aproximadamente el 20 por 100 del crecimiento del PIB. A partir del año 2008 el fuerte impacto en el sector de la construcción de la contracción del crédito inmobiliario se concreta en fuertes ajustes de cantidades en el sector, el número de viviendas iniciadas alcanza su mínimo histórico en el año 2014, con más de 50.000 unidades de vivienda iniciadas (Gráfico 1). Este reajuste no es homogéneo en todo el territorio español, las Comunidades Autónomas (CCAA) con mayores disminuciones son las localizadas en la costa mediterránea (Murcia, Comunidad Valenciana, Islas Baleares, Canarias, Andalucía y Cataluña), así como en el centro y norte (Madrid, Castilla León, La Rioja, Galicia, Asturias).

\section{GRÁFICO 1}

VIVIENDAS INICIADAS EN ESPAÑA, 1986-2014

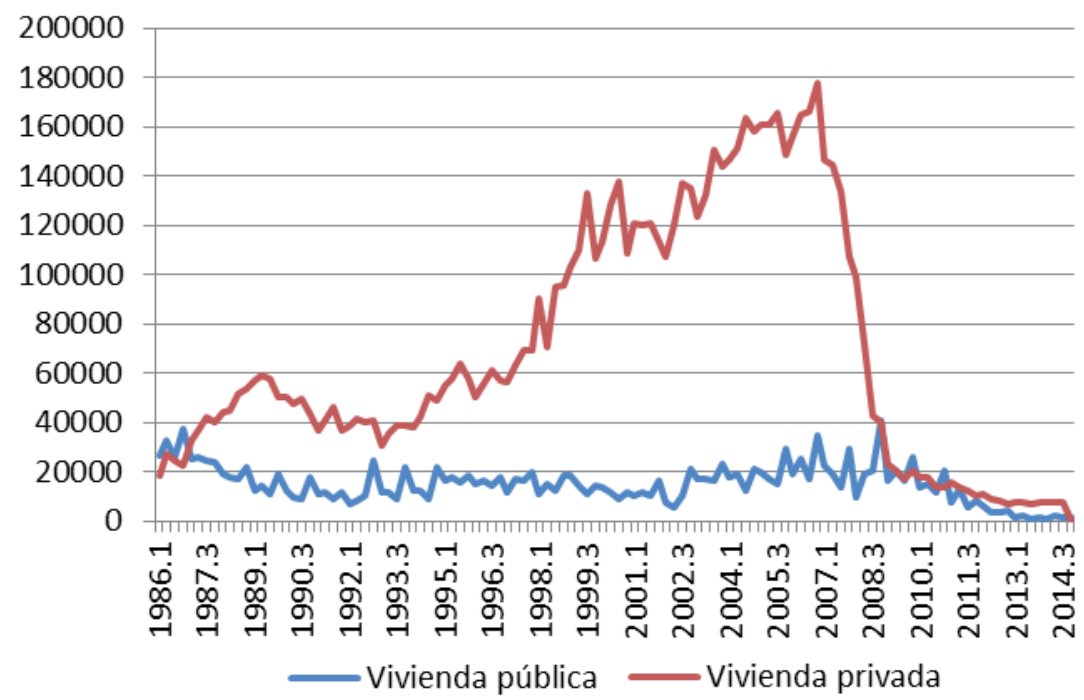

FUENTE: Ministerio de Fomento. 
Este ciclo de edificación residencial se caracteriza por una significativa reducción en los niveles de construcción de vivienda pública ${ }^{l}$ provistos en España. Los periodos de máxima expansión se han visto acompañados de una caída en el número de viviendas de protección pública iniciadas (Gráfico 1), con un déficit de unidades que generó una llamada de atención por parte de la sociedad hacia los poderes públicos, que comenzó a aparecer a medida que la accesibilidad de los hogares de primera entrada al mercado de vivienda se reducía como resultado del aumento de precios y de la tensión en la demanda de alquiler, fechados ambos, aproximadamente, entre los años 2002 y 2003.

Las características propias de la política de vivienda en España hacen que existan disparidades territoriales en el ciclo de vivienda pública. En España existe un sistema mixto de provisión en el que participa la Administración del Estado, las Comunidades Autónomas y los ayuntamientos. El gobierno central es el encargado, entre otros aspectos, de la coordinación de la política de vivienda. Las Comunidades Autónomas tienen la potestad de modificar y complementar la política del estado central con sus propios recursos y son responsables de establecer las regulaciones en materia de vivienda y suelo así como de gestionar el parque de alquiler autonómico subvencionado, el stock de vivienda pública, las concesiones de ayudas y establecer el control de las inversiones en vivienda. Por último, los ayuntamientos son los responsables de la planificación urbana y gestión del suelo en el proceso de urbanización.

Todo ello unido a la existencia de distintos modelos residenciales en las Comunidades Autónomas, hace que la intensidad y caracterización del ciclo de vivienda pública y privada difiera entre autonomías. El Cuadro 1 muestra el porcentaje en términos anuales de viviendas públicas iniciadas sobre el total de viviendas iniciadas por CCAA y agrupados en periodos. Los valores porcentuales confirman un comportamiento muy diferenciado entre las distintas CCAA, y dentro de éstas entre las distintas provincias que las integran. Las cifras que reflejan el auge del ciclo expansivo muestran disparidades importantes, Aragón, País Vasco, Navarra y la Comunidad de Madrid incorporan al ciclo de vivienda una proporción de vivienda pública superior al 30 por 100, Islas Baleares, Islas Canarias, Cataluña, Comunidad Valenciana y Región de Murcia presentan un peso porcentual de las viviendas públicas iniciadas inferior al 10 por 100 del total. Para el conjunto de España el peso se concreta en un 11 por 100 sobre el total de viviendas iniciadas.

\footnotetext{
${ }^{1}$ En este artículo, se entiende por vivienda pública a todas aquellas unidades que han recibido ayuda pública de algún tipo y se han sometido al control de la regulación, independientemente de su uso, clasificación en los distintos planes, o nivel de ayuda. En este sentido, el artículo define como ciclo público al ciclo de edificación de vivienda pública, y al ciclo privado el de edificación de vivienda libre, ambos medidos a partir de las estadísticas de viviendas iniciadas.
} 


\section{CUADRO 1 \\ EVOLUCIÓN DEL PORCENTAJE DE VIVIENDA PÚBLICA INICIADA SOBRE EL TOTAL DE VIVIENDA INICIADA (COMUNIDADES AUTÓNOMAS) \\ (En \%)}

\begin{tabular}{|l|c|c|c|c|c|c|c|c|c|}
\hline & España & Madrid & $\begin{array}{c}\text { Astu- } \\
\text { rias }\end{array}$ & Alicante & $\begin{array}{c}\text { Guada- } \\
\text { lajara }\end{array}$ & Cáceres & $\begin{array}{c}\text { Valla- } \\
\text { dolid }\end{array}$ & Vizcaya & $\begin{array}{c}\text { Zara- } \\
\text { goza }\end{array}$ \\
\hline $1986-1992$ & 49,94 & 61,41 & 197,15 & 29,19 & 34,97 & 280,35 & 357,51 & 736,49 & 255,84 \\
\hline $1993-1999$ & 25,67 & 24,68 & 32,28 & 22,70 & 27,04 & 74,42 & 8,52 & 23,32 & 11,24 \\
\hline $2000-2007$ & 12,08 & 30,17 & 10,24 & 9,90 & 6,74 & 21,33 & 13,58 & 42,29 & 24,17 \\
\hline $2008-2014$ & 58,69 & 190,60 & 51,66 & 37,65 & 26,67 & 47,91 & 46,45 & 112,80 & 125,27 \\
\hline
\end{tabular}

FUENTE: Ministerio de Fomento.

La situación en la cual las condiciones de mercado en expansión reducen los incentivos a la construcción de vivienda pública no es nueva en España, y parece haberse repetido, al menos, durante tres periodos más, 1975-78, 1988-90, 1999-2001 (Taltavull, 2003). Todos ellos son fases de crecimiento final previo a una recesión, lo que sugiere la existencia de una interrelación entre ambos ciclos, un efecto de arrastre a la baja de las viviendas públicas al invertirse la tendencia y un descenso del ciclo de edificación pública sin aparente justificación siguiendo los principios económicos de funcionamiento del mercado.

No obstante, si se considera que la provisión de vivienda pública se efectúa para para reducir el problema de acceso a vivienda de los segmentos de población con menores recursos, sería razonable que su presencia se redujese en los momentos en que el mercado es más eficiente en la provisión de viviendas, o dicho con otras palabras, en aquellos periodos en los que la combinación de condiciones de demanda favorezca el acceso al mercado ${ }^{2}$. Por otro lado, una vez invertido el ciclo de edificación, y cuando las condiciones de mercado no son favorables al acceso y la demanda de primera vivienda se acumula, podría ocurrir lo contrario, es decir, que los proyectos originalmente concebidos como privados se reorientasen a públicos, apareciendo una reacción contracíclica. La exposición de motivos de las distintas políticas de viviendas en España desde los sesenta parece sugerir que este fenómeno ha estado presente en las distintas décadas, aunque no ha sido contrastado empíricamente. La habilidad de la política de vivienda consistiría en identificar estos momentos para interactuar con el ciclo, de forma que su efecto contra cíclico alcanzase los objetivos deseados. Desde esta perspectiva, la política de vivienda no se vería arrastrada por el ciclo económico sino que se independizaría de él en los momentos necesarios.

Sin embargo, las medidas recientes adoptadas en los planes de vivienda desde principios del siglo XXI obligando a cuotas de producción de unidades públicas en

${ }^{2}$ Las condiciones de demanda, tanto aquellos con efectos permanentes, como son las demográficas, renta o financiación, como las que tienen efectos transitorios, como los costes de transacción. Los determinantes de demanda están bien explicados por la teoría inmobiliaria (DI PASQUALE y WHEATON, 1996). 
función de las libres, insertan un automatismo indiciando la edificación pública a la privada que tendría el efecto contrario, arrastrando a la baja en periodo de caída en la construcción y al alza en periodo de expansión. El sobredimensionamiento de la oferta que existiría si la política tuviese éxito y la escasez en época recesiva podría tener impacto sobre la eficiencia de este mercado, aunque no hay evidencia sobre el hecho de que estos efectos hayan aparecido.

Este artículo analiza el modelo de interrelación entre las viviendas públicas y privadas en España, esto es, la existencia de un efecto crowding out entre el ciclo público y privado de vivienda. En concreto se define y mide el modelo de comportamiento temporal entre ambos ciclos en términos agregados en el territorio nacional, y entre distintas Comunidades Autónomas de España.

El desarrollo del trabajo se organiza como sigue: en la sección 2 se muestra una revisión de la literatura; en la 3 se describen, en un análisis exploratorio inicial, los ciclos de edificación público y privado; en la sección 4 se presenta la estrategia de modelización, la definición de crowding out, la descripción de los datos utilizados y los resultados del ejercicio empírico; se concluye con la sección 5.

\section{Revisión de la literatura}

La notable expansión del sector de construcción es una característica común a todas las economías desarrolladas desde mediados de los noventa hasta la actualidad. Los distintos estudios que analizan este ciclo ponen de manifiesto la relevancia de las características locales para determinar las razones de su crecimiento (Quigley, 2007; Quigley et al., 2008; Ball, 2011; Ball et al., 2010). El análisis empírico resalta el papel de finanzas, innovaciones monetarias y mercado de capitales (Muelbauer, 2007; Ortalo-Magne y Rady, 2006), así como de los cambios financieros, liquidez e impacto de la política monetaria en la financiación de la vivienda (Taltavull y White, 2012).

No obstante, a pesar de la importancia de la inversión pública en vivienda sobre el stock del mercado de vivienda y su precio, hay muy pocos estudios que aborden las interacciones entre el ciclo público y privado de vivienda analizando el efecto crowding out entre ambos.

Los primeros análisis surgen en finanzas y economía de la vivienda con los trabajos de Swan $(1973)$ y Murray $(1983,1999)$ que utilizan una estructura de ofertademanda standard mediante la estimación con variables instrumentales. En estudios posteriores, los modelos de vivienda en su forma reducida son considerados como alternativa a los modelos estructurales (Malpezzi y Vandell, 2002; Sinaí y Waldfogel, 2002). Malpezzi y Vandell (2002) estiman un modelo transversal para la variación de unidades de vivienda per cápita en el ámbito territorial de Estados Unidos. La urbanización, la estructura por edad de la población y el crecimiento demográfico explican gran parte de la variación en la oferta de vivienda. Sin embargo, en la mayor parte de los países, la oferta está formada por viviendas que construyen la ini- 
ciativa pública y la privada, y la relación entre ambos ciclos de edificación ha sido poco estudiada. En esencia, los trabajos que lo abordan defienden que existen efectos cruzados, de sustitución o expulsión entre las viviendas construidas de promoción privada y pública.

Cuando se considera que las decisiones en un ámbito de la economía tomadas desde el sector público afectan a la misma actividad desarrollada por la iniciativa privada, se produce el efecto crowding out (cuando hay expulsión) o crowding in (cuando hay un incentivo a dicha actividad). Este concepto ha sido normalmente aplicado al análisis de los impactos de la política monetaria o fiscal sobre la economía aunque también a sectores donde la presencia de la intervención pública es importante. Han sido conocidos como el hidden costs of rewards, costes ocultos de la ayuda pública (Frey y Jergen, 2000).

En esencia, el efecto crowding out actuaría como aparece en el Esquema 1 (parcialmente tomado de Yuan y Amori, 2014:4324). En un mercado residencial con vivienda privada mayoritaria, la intervención pública construyendo nuevas unidades (más accesibles, con menores precios) tiene el efecto de amentar la oferta pero también de desincentivar la producción de unidades al precio de mercado. Esto haría que el aumento efectivo de viviendas construidas $\left(\mathrm{V}_{1}-\mathrm{V}_{0}\right)$ sea menor que el potencial $\left(\mathrm{V}_{2}-\mathrm{V}_{0}\right)$ como resultado de la intervención, y que la oferta de nuevas unidades lo sea de viviendas públicas.

Las causas de que estos desplazamientos ocurran en el mercado de la vivienda podrían ser dos (Murray, 1999). La primera sería la competencia entre promotores

\section{ESQUEMA 1}

\section{EFECTO CROWDING OUT ENTRE VIVIENDAS PÚBLICAS Y PRIVADAS}

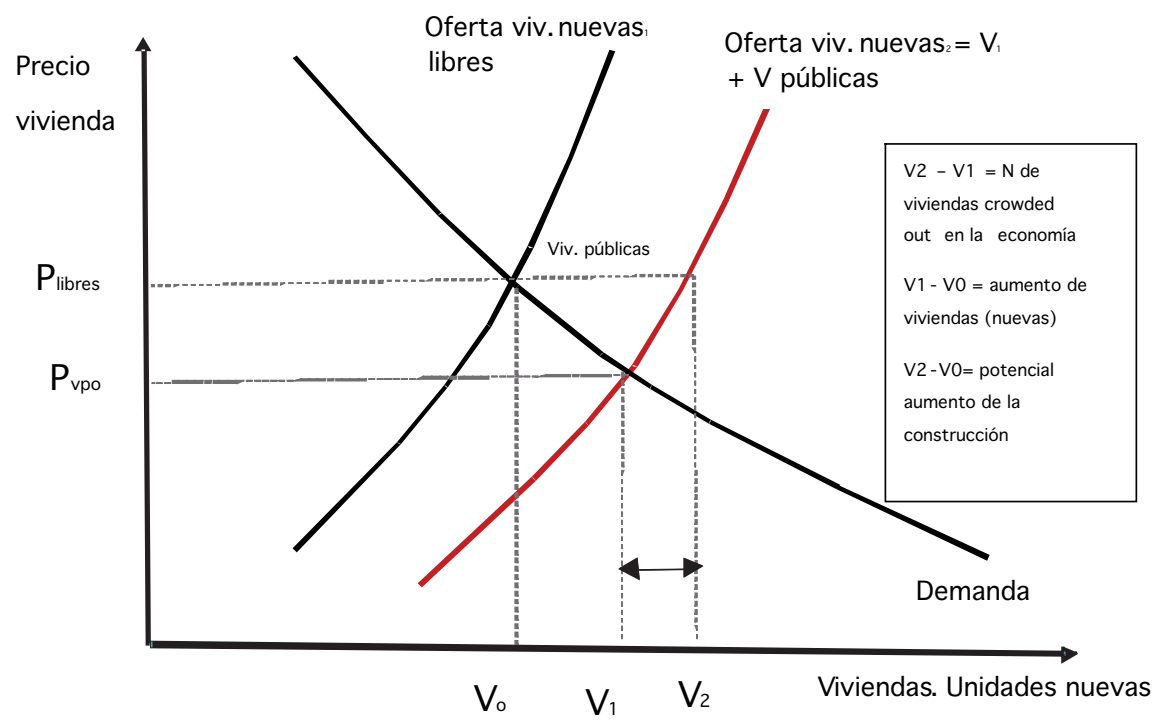


privados y públicos por los créditos hipotecarios para financiar la construcción. En la medida en que los recursos financieros son escasos, una política pública de vivienda agresiva absorbería una parte importante de los recursos del sistema financiero y reduciría los destinados a los promotores privados. La segunda sería la competencia por la demanda. Dados los niveles de calidad y condiciones técnicas mínimas que deben cumplir las viviendas en España derivadas de las normativas, su diseño y localización las hacen ser bienes sustitutivos de las viviendas privadas. Además, como su precio es fijado a niveles menores que los de mercado (son precios tasados que se determinan anualmente)la demanda tiende a absorber, primero, las unidades públicas y después a las privadas, ejerciendo así, la edificación pública, el efecto de expulsión definido.

La identificación de la existencia de efectos crowding no es sencilla, como muestra una reducida evidencia empírica a la que se hace referencia más adelante, y con una larga disparidad en los resultados. La mayoría de estos trabajos optan por examinar las posibles relaciones causales entre los ciclos, de manera que si la relación dinámica entre ambas actividades es estadísticamente significativa, se considera que existe evidencia que respalda el efecto crowding entre provisión pública y privada. Entorno a esta visión, los trabajos buscan evidencia empírica (completa o parcial) sobre la existencia de efectos de expulsión. Así, Sinaí y Waldfogel (2002), analizan como la existencia de programas públicos (locales) de vivienda afectan al equilibrio del mercado en el contexto de divisiones territoriales del mercado local. Sus resultados indican la existencia de un efecto sustitución en el mercado.

Murray (1999) y Evenson (2004) utilizan la metodología de series temporales con objeto de analizar la dinámica a corto y largo plazo. Murray (1999) utiliza herramientas de cointegración para el análisis de las series de stocks de vivienda pública y libre e ingreso real. Sus resultados indican la no existencia de un efecto desplazamiento entre vivienda pública de bajos ingresos y vivienda libre, mientras que la vivienda pública dirigida a familias de ingresos medios no presenta una aportación significativa al parque de vivienda. Evenson (2004) y Yuan y Hammon (2014) también utilizan la metodología VAR para caracterizar la respuesta dinámica del precio y stock de vivienda ante un incremento de la demanda consecuencia de variaciones en el nivel de empleo.

Chul-In Lee (2006) incorpora en el análisis la metodología de datos panel para analizar las interacciones dinámicas entre vivienda de alquiler privado y su contrapartida pública en el mercado de Corea en el periodo 1988-2003. A partir de la modelización VAR, se examina la eficacia de los programas de apoyo de vivienda para unidades familiares de renta baja. El análisis empírico pone de manifiesto la existencia de un efecto crowding out entre la inversión en vivienda pública y privada.

De forma similar, Nordvik (2006) analiza cómo el parque de viviendas en los municipios noruegos se ve afectado por la intervención pública en dicho mercado. El análisis empírico, de corte transversal, muestra qué unidades adicionales de vivienda pública incrementan el parque total de viviendas. Análogamente, Eriksen y Rosenthal (2010) efectúan un análisis de los efectos desplazamiento entre unidades de vivienda 
del sector público y privado a partir de la agregación de datos transversales en distintos clusters geográficos y sus resultados muestran la existencia de un efecto crowding en el sector del alquiler de mercado de la vivienda.

Yuan y Hamori (2014), utilizando datos de panel, examinan las interacciones dinámicas entre la construcción de vivienda accesible (pública) y no accesible (privada) en China. Encuentran que la respuesta de las segundas ante las construcción de las primeras cambia en el tiempo produciéndose un efecto crowding out, que no es simétrico. El efecto deja de ocurrir cuando los precios de ambas viviendas están próximos o son iguales.

Los distintos estudios arrojan resultados parciales, no siempre concluyentes acerca de la existencia del efecto expulsión entre el ciclo público y privado de vivienda; en función del territorio analizado y las medidas de política pública empleadas, los resultados varían. El impacto de las mismas sobre el mercado dependerá de elasticidades precio e ingreso sobre la demanda y la oferta, y pueden provocar cambios relevantes en la configuración de la zona en la que se construyen las viviendas públicas (Malpezzi y Vandel, 2002; Whitehead, 2003).

El mercado de la vivienda en España muestra particularidades propias que sugieren que este efecto puede ser importante. España es un país de propietarios de viviendas. Según los datos del Censo de Población y Vivienda 2011, el 79 por 100 de las viviendas principales españolas están ocupadas en régimen de propiedad, un 13,5 por 100 pertenece al sector de alquiler y un 7,5 por 100 pertenece a la categoría «cedida gratis» u «otros». Las viviendas en régimen de alquiler son propiedad principalmente de propietarios privados individuales, siendo la cuota del alquiler social o subvencionada del sector público muy limitada. La vivienda pública en España es principalmente en régimen de propiedad, con rasgos distintivos por cada Comunidad Autónoma y problemáticas intrínsecas que han sido abordadas a través del diseño de las políticas de vivienda y regulaciones paralelas. En el caso de España, la vivienda pública se ha venido articulando asociada al ciclo de edificación privada, bien a partir de la fijación de cuotas obligatorias de edificación pública (arriba mencionadas, que en algunas Comunidades Autónomas son muy importantes, como en Madrid con el 50 por 100 o en la Comunidad Valenciana, con el 30 por 100), o bien a través de incentivos directos a los promotores privados para la edificación de viviendas públicas (tras la fijación de objetivos concretos en cuanto a número de viviendas a iniciar y acuerdos contractuales con los promotores). Este sistema ha hecho que la decisión última de comenzar una promoción de vivienda pública pueda encontrarse fuertemente asociada a la decisión de inversión privada, es decir, fuerza al promotor de vivienda libre a tener en cuenta la construcción conjunta o ulterior de unidades públicas, si quiere comenzar el proyecto ${ }^{3}$. No hay evidencia empírica sobre el sentido de esta relación, pero es razonable pensar que ambas decisiones se vean afectadas no siempre en la misma dirección, pudiendo variar su influencia en función

\footnotetext{
${ }^{3}$ Esta cuestión es relevante ya que la normativa pública está obligando a los planes de inversión privadas interfiriendo en sus parámetros básicos, como rentabilidad o estimación de demanda.
} 
de las normas territoriales de política de vivienda. Esta interacción entre construcción de vivienda pública y libre y cómo la construcción de un tipo de vivienda afecta a la otra, es la relación que se analiza en este trabajo.

La existencia de fuertes diferencias en la definición y aplicación de la política entre territorios españoles consecuencia de distintos modelos residenciales y de políticas de vivienda diversas, implica que el análisis a nivel agregado no es suficiente para comprender el fenómeno de crowding (in o out) entre el ciclo público y privado de vivienda español, y es necesario observar esa reacción a un nivel geográfico más detallado. Por esta razón, el análisis se lleva a cabo desagregado por algunas provincias.

\section{Caracterización del ciclo residencial. Las fórmulas de provisión}

La evolución de los ciclos residenciales de vivienda pública y libre en España es dispar (Gráfico 2). La elevada variabilidad en los precios de las viviendas, la existencia de distintos modelos residenciales y la diversa actuación del sector público en cada Comunidad Autónoma constituyen algunas de las razones de dicha heterogeneidad.
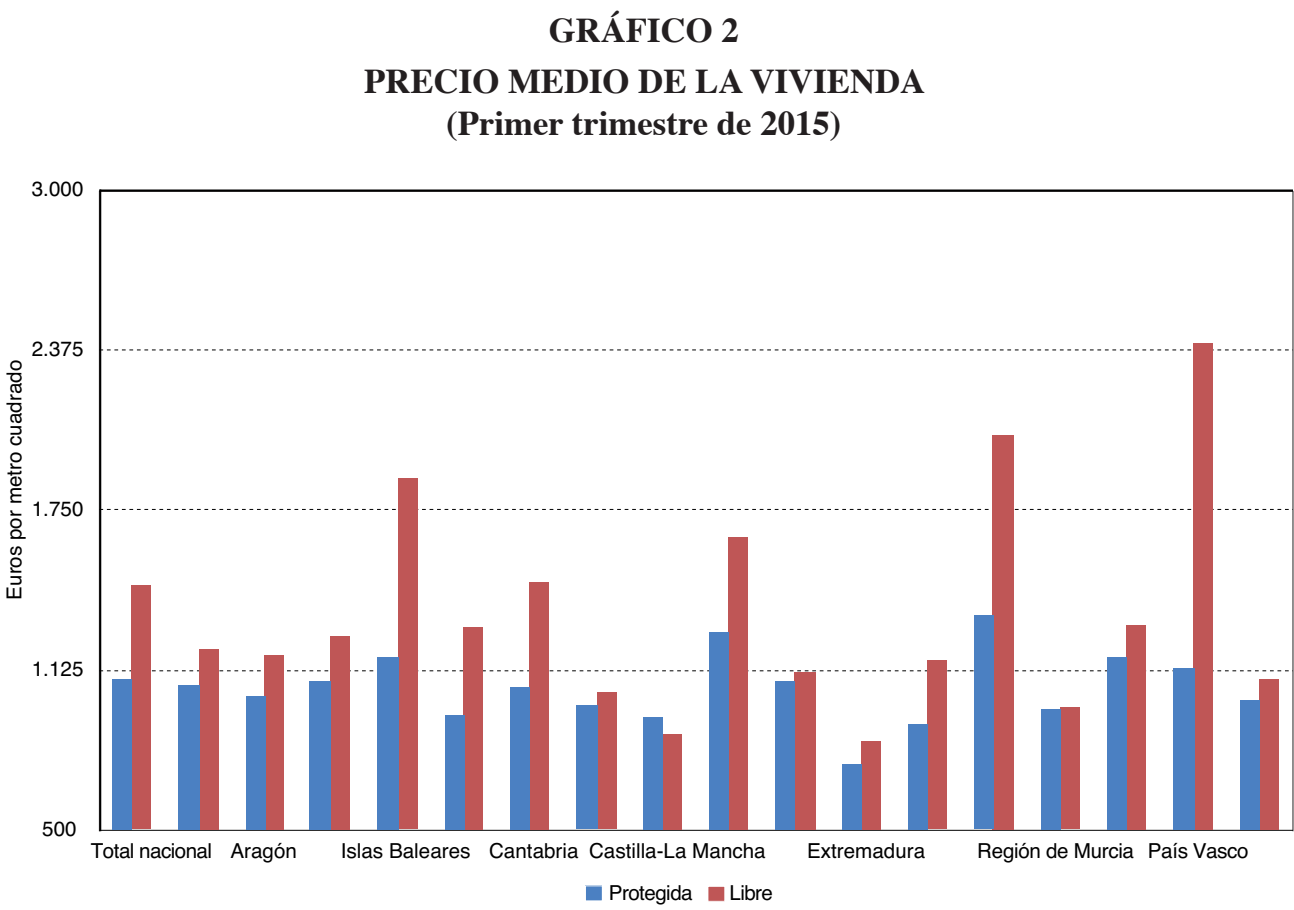

FUENTE: Ministerio de Fomento. 
La división territorial en provincias que forma parte de la estructura administrativa española (17 Comunidades Autónomas más dos Ciudades Autónomas) constituye la unidad básica de este análisis más desagregada posible a partir de los datos existentes. En España existen un total de 50 provincias, de las cuales se han seleccionado ocho de ellas para el análisis: Madrid, Asturias, Alicante, Guadalajara, Cáceres, Vizcaya, Zaragoza y Valladolid. La elección se ha realizado atendiendo al nivel de precios de la vivienda libre y protegida ${ }^{4}$ de manera que se tuviese representación de todos los cuartiles de la distribución.

Así se han tomado las provincias pertenecientes a dos regiones que caen en el cuartil de precios mayores (cuarto cuartil en la distribución ordenada de precios, Comunidad de Madrid y País Vasco), dos con los precios menores (primer cuartil, Extremadura y Castilla-la Mancha), y dos respectivamente que caen en el segundo y tercer cuartil de la distribución (Castilla y León, Aragón, Comunidad Valenciana y Principado de Asturias, en este orden) representan el segundo y tercer cuartil. La selección de las provincias, cuando hay más de una, se realiza siguiendo el mismo criterio.

La evolución de los ciclos de vivienda en cada uno de los ámbitos territoriales analizados se recoge en el Gráfico $3^{5}$. En todos ellos se constata un empuje similar de la vivienda libre frente al ciclo de unidades públicas iniciadas.

Los ámbitos territoriales elegidos constituyen ejemplos dispares del mercado de la vivienda en España. En todos los casos, las provincias seleccionadas poseen variables que explicarían un comportamiento distinto de sus factores de demanda $y$, por ende, de la intensidad con la que actúan en el ajuste del mercado ${ }^{6}$; el peso relativo del mercado de vivienda y ritmo de actividad en edificación pública y privada también difieren entre regiones.

Como se observa en el Gráfico 3, el rol que los tres ciclos públicos ejercen sobre el total de edificación parece ser dispar en Madrid, Cáceres y Vizcaya. En este caso, las tres provincias cuentan con una proporción de vivienda pública iniciada respecto del total muy superior al resto de las estudiadas, mientras que Alicante y Guadalajara presentan el peso más bajo. En el total del periodo considerado el peso de la vivienda pública en Cáceres se concreta en un 31,36 por 100, frente al 10,97 por 100 de Guadalajara, mientras que en Madrid la vivienda pública iniciada creció con intensidad desde niveles prácticamente nulos en 2003 y superó el máximo histórico de edificación del periodo en 2008, en Asturias esta reacción se dio en 2006; aunque con un nivel sensiblemente inferior al alcanzado en 1985, el máximo histórico asturiano se localizó en el tercer trimestre de 2009 con 1.838 unidades de vivienda protegida. En Alicante el repunte de la vivienda pública se produjo en 2000 y tras la fuerte caída en la edificación en 2008 también cayeron los niveles de producción de vivienda pública como en el resto del territorio.

\footnotetext{
${ }^{4}$ El análisis de selección puede ser solicitado a los autores.

5 Se utilizan las viviendas iniciadas en el análisis, siguiendo la literatura sobre ciclos residenciales que usan este indicador como la variable de referencia de la inversión residencial (SMITH et al., 1988).

${ }^{6}$ Aunque la oferta está más estudiada (TALTAVULL, 2012, 2014), hay reducidos análisis sobre los condicionantes de demanda residencial en España a nivel desagregado.
} 


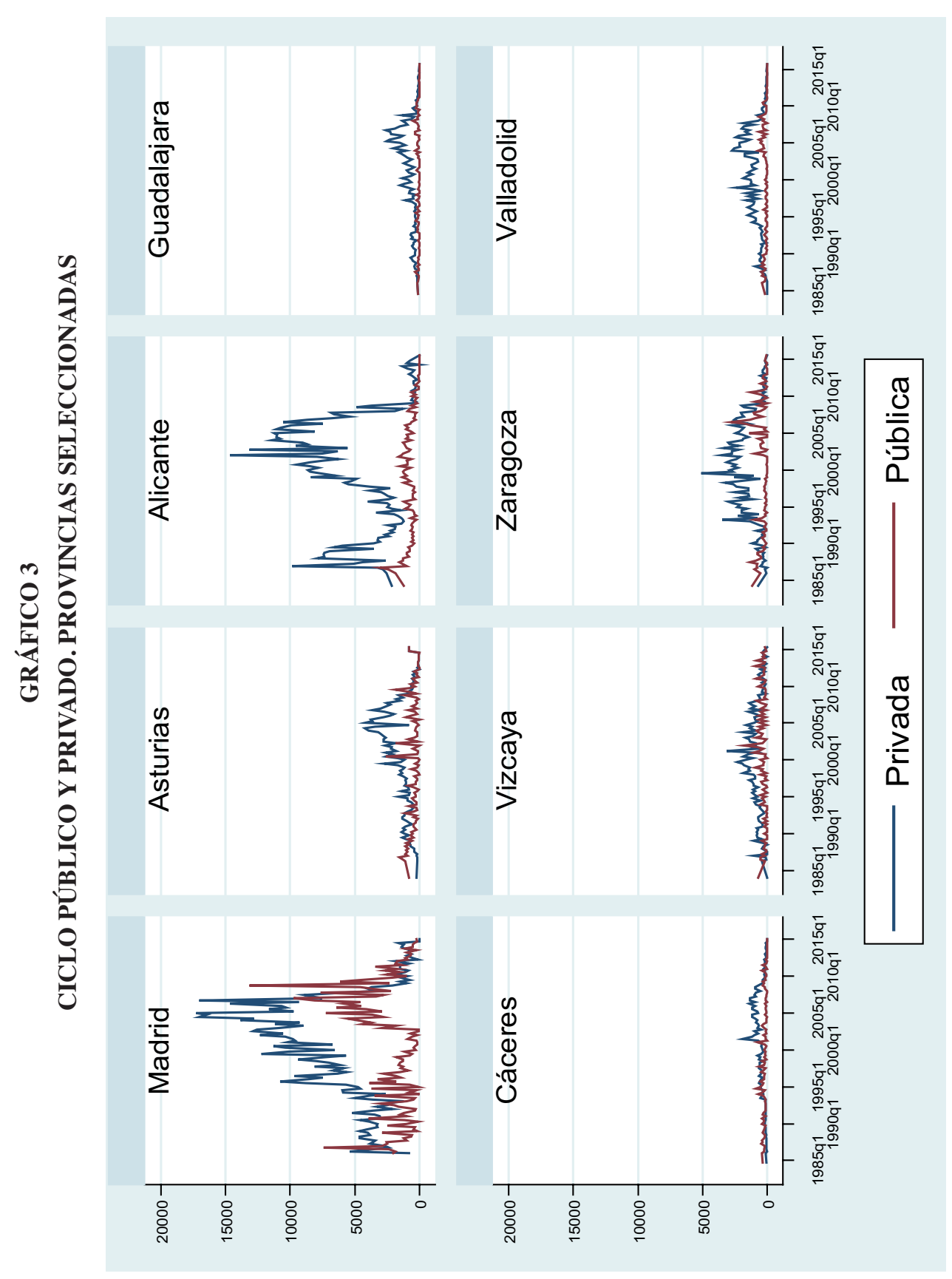

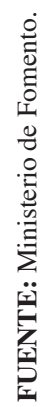


En los ocho ámbitos territoriales analizados, los procedimientos seguidos para la provisión de la política de vivienda son inicialmente similares, aunque el proceso de transmisión final de los incentivos para la construcción a los promotores, más complejo, se realiza de forma indirecta y diferente por regiones, a través de estímulos tanto a la oferta como a la demanda. La posibilidad de obtener financiación en condiciones preferenciales o suelo público para desarrollar, y la concesión de créditos preferenciales a los compradores que satisfagan las condiciones previstas en el plan, constituyen ejemplos, en uno y otro sentido, de la intensidad en la intervención pública y los incentivos mencionados. La flexibilidad en los sistemas varía entre provincias; por un lado, aquellas en donde la provisión de vivienda pública es unimodal (asignada a hogares que cumplan ciertos requisitos) y, por otro, aquellos sistemas mixtos en los la construcción de vivienda libre y vivienda pública es simultánea ${ }^{7}$.

El proceso de implementación de la política de vivienda es variado dado que es el promotor privado quien decide el momento y localización de la promoción de la vivienda pública. Ello significa que el seguimiento de la gestión y aplicación de la intervención pública no constituye una labor sencilla. El hecho de que sea el promotor privado quien decida entre construir vivienda libre o pública ha podido explicar el dominio de las primeras en momentos expansivos del ciclo y con condiciones de demanda favorables, y el consecuente abandono de la edificación pública anteriormente señalado, en otros momentos. También justifica el retorno a la edificación pública cuando las condiciones del mercado empeoran.

\section{La estrategia de modelización empírica, datos y resultados de los modelos}

La identificación del efecto crowding entre provisión pública y privada en España se realiza, en este trabajo, contrastando los efectos dinámicos existentes entre ambos ciclos de edificación con especial atención a los periodos de influencia entre ambos y a la dirección de la relación. Es decir, se pretende contrastar si el ciclo de viviendas de un tipo (pública, denominada en adelante «protegida» por la especificación de las estadísticas en España, o privada) arrastra o se ve afectado por el ciclo del otro tipo. Es decir:

$$
\Gamma\left(\text { Vpriv }_{t-i}\right) \Leftrightarrow \Phi\left(\text { Vprot }_{t-i}\right) \quad \forall t=1, \ldots, T \quad \forall t \geq 0
$$

siendo Vpriv ${ }_{t}$ las viviendas iniciadas libres en el momento $t$, Vprot $t_{t}$ las viviendas iniciadas protegidas, $\Phi$ y $\Gamma$ son matrices de parámetros que relacionan ambas variables en distintos momentos del tiempo y $t-i$ hace referencia al periodo temporal.

\footnotetext{
${ }^{7}$ Agradecemos a un evaluador el especificar este concepto.
} 
Como se contrasta la relación biunívoca, el ejercicio captura la reacción simultánea de ambas construcciones, lo que puede plasmarse en las expresiones [2] y [3].

$$
\begin{aligned}
& \operatorname{Vpriv}_{t}=\alpha_{1}+\sum_{i=1}^{n} \beta_{1 i} \text { Vprot }_{t-i}+\varepsilon_{i} \\
& \operatorname{Vprot}_{t}=\alpha_{2}+\sum_{i=1}^{n} \beta_{2 i} \text { Vpriv }_{t-i}+\mu_{i}
\end{aligned}
$$

donde los $\alpha$ y $\beta_{i}$ son los parámetros que capturan las relaciones entre los ciclos y $\varepsilon, \mu$ son términos de error.

Se utilizan las series temporales ${ }^{8}$ de los indicadores de vivienda iniciadas LIBRES y PROTEGIDAS elaborados por el Ministerio de Fomento para el periodo comprendido entre el primer trimestre de 1986 y el mismo de 2015. La muestra está integrada por 117 observaciones de frecuencia trimestral cuyos estadísticos básicos se encuentran en el Cuadro 2.

Formalmente se sigue la metodología de Johansen (1992). En primer lugar, se contrasta la hipótesis de estacionariedad mediante test de Dickey-Fuller Aumentado (ADF), y se comprueba que las variables LIBRES y PROTEGIDAS se caracterizan como estacionarias o no estacionarias e integradas de primer orden en los diferentes ámbitos territoriales. La hipótesis nula a contrastar, la existencia de raíces unitarias, es rechazada ${ }^{9}$ en niveles para la variable PROTEGIDAs en Alicante, Guadalajara y Zaragoza y en primeras diferencias en el resto de las variables ( $p$-valor $=0,01)$. Los resultados se encuentran en el Cuadro $3^{10}$.

La interdependencia entre las series consideradas inicialmente endógenas mediante la estimación de un VAR $^{11}$ visualiza cada variable, LIBRES y PROTEGIDAS, como una función del pasado de ambas. A partir de la determinación del retardo

${ }^{8}$ Dado que el fenómeno se observa a través de series temporales, la definición precisa de su relación en el tiempo dependerá de los atributos estadísticos de las series, por lo que se contrasta, primero, la existencia de raíces unitarias y su orden de integración, para, luego, realizar los contrastes de cointegración que permitan definir el modelo empírico final que describa adecuadamente las relaciones contemporáneas y dinámicas entre sus componentes. El análisis de la estacionariedad de cada una de las series, la determinación de posibles relaciones de cointegración mediante el enfoque de máxima verosimilitud de JOHANSEN (1988, 1992) y la estimación del proceso dinámico de ajuste a la relación de equilibrio a largo plazo mediante un MCE, constituye la pauta a seguir. La ventaja del enfoque de cointegración consiste en que permite una aproximación a la especificación del modelo causal y al conocimiento real del fenómeno económico en etapas avanzadas de su análisis (AMEMIYA, 1980).

${ }^{9}$ El rechazo de la relación de cointegración en las provincias mencionadas implica que las relaciones entre ambos ciclos no tiene efectos permanentes y se dan en el corto plazo. En estos casos, la identificación del crowding out se produce con efectos de corto alcance o, dicho con otras palabras, que puede cambiar en función de las decisiones tomadas que no siguen una pauta estable en el tiempo.

${ }^{10}$ En todo el ejercicio se ha controlado por cambio estructural. Se han realizado los análisis en ausencia de tales cambios para luego estimar los breaks en las series de residuos e identificar la existencia de cambios estructurales en cada una de ellas. Una vez aislado el cambio, e identificado si son temporales (corto plazo) o permanentes, han sido introducidos en forma de dummies en los ejercicios.

${ }^{11}$ Dicha modelización evita las imposiciones derivadas de la estimación e identificación de un modelo econométrico y permite la especificación de modelos que reflejen lo más fielmente posible las regularidades empíricas e interacciones entre las variables objeto de análisis (SIMS, 1980). 


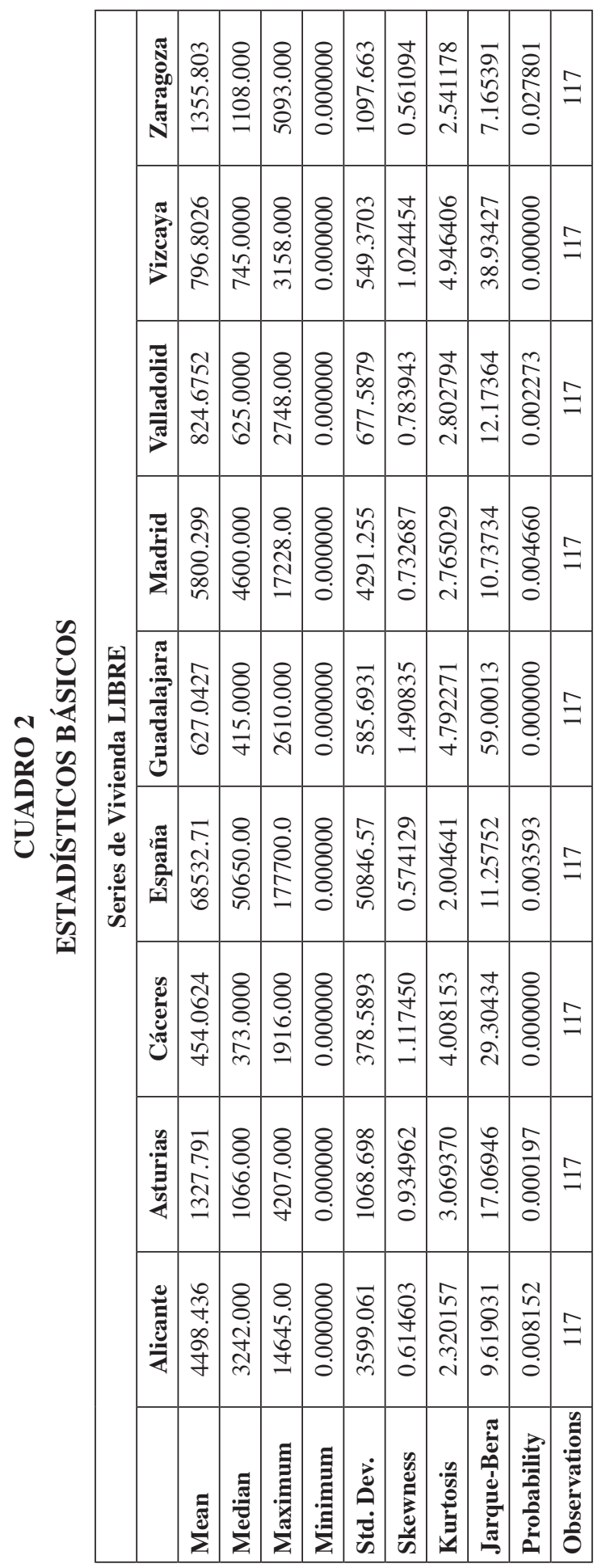




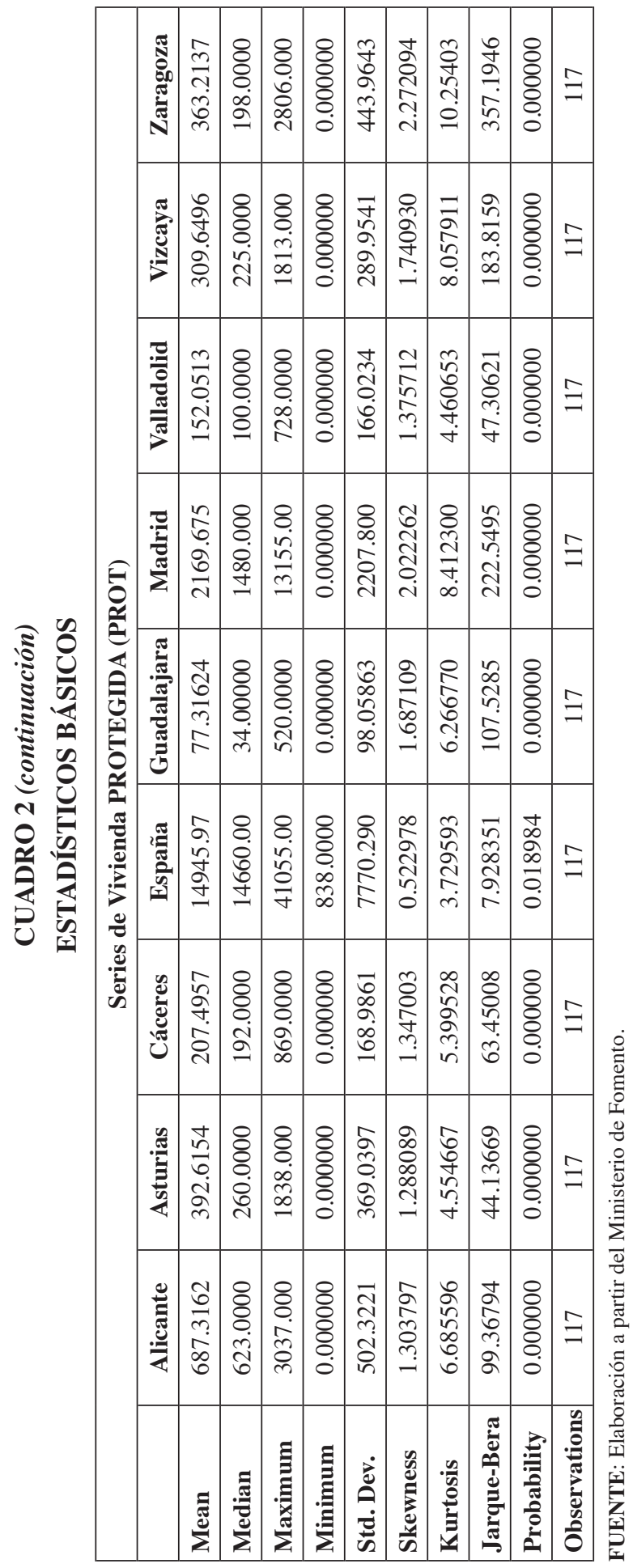




\section{CUADRO 3 \\ TEST DE RAÍCES UNITARIAS}

\begin{tabular}{|c|c|c|c|c|c|c|}
\hline & & \multicolumn{4}{|c|}{ ADF-Augmented Dickey-Fuller test statistic* } & \multirow{2}{*}{$\begin{array}{c}\text { Orden de } \\
\text { integración }\end{array}$} \\
\hline & & Nivel & p-valor & $\begin{array}{c}1 \\
\text { Diferencias } \\
\end{array}$ & p-valor & \\
\hline \multirow{2}{*}{ Alicante } & Libres & $-0,953$ & 0,3022 & $-10,364$ & 0,000 & $\mathrm{I}(1)$ \\
\hline & Protegidas & $-3,781$ & 0,0002 & - & 0,000 & $\mathrm{I}(0)$ \\
\hline \multirow{2}{*}{ Cáceres } & Libres & $-1,394$ & 0,1512 & $-15,293$ & 0,0000 & $\mathrm{I}(1)$ \\
\hline & Protegidas & $-1,796$ & 0,0690 & $-10,627$ & 0,000 & $\mathrm{I}(1)$ \\
\hline \multirow{2}{*}{ Guadalajara } & Libres & $-0,973$ & 0,2940 & $-13,655$ & 0,0000 & $\mathrm{I}(1)$ \\
\hline & Protegidas & $-3,819$ & 0,000 & - & - & $\mathrm{I}(0)$ \\
\hline \multirow{2}{*}{ Zaragoza } & Libres & $-0,996$ & 0,2848 & $-10,058$ & 0,0000 & $\mathrm{I}(1)$ \\
\hline & Protegidas & $-2,105$ & 0,0345 & $-12,913$ & 0,0000 & $\mathrm{I}(0)$ al $5 \%$ \\
\hline \multirow{2}{*}{ ESPAÑA } & Libres & $-1,205$ & 0,2079 & $-2,900$ & 0,0040 & $\mathrm{I}(1)$ \\
\hline & Protegidas & $-1,915$ & 0,0533 & $-5,808$ & 0,0000 & $\mathrm{I}(1)$ \\
\hline \multirow{2}{*}{ Asturias } & Libres & $-0,982$ & 0,290 & $-14,244$ & 0,0040 & $\mathrm{I}(1)$ \\
\hline & Protegidas & $-2,387$ & 0,0671 & $-14,185$ & 0,0000 & $\mathrm{I}(1)$ \\
\hline \multirow{2}{*}{ Madrid } & Libres & $-1,090$ & 0,2487 & $-17,816$ & 0,0000 & $\mathrm{I}(1)$ \\
\hline & Protegidas & $-1,657$ & 0,0921 & $-11,277$ & 0,0000 & $\mathrm{I}(1)$ \\
\hline \multirow{2}{*}{ Valladolid } & Libres & $-1,225$ & 0,2013 & $-16,161$ & 0,0000 & $\mathrm{I}(1)$ \\
\hline & Protegidas & $-1,638$ & 0,0954 & $-10,619$ & 0,0000 & $\mathrm{I}(1)$ \\
\hline \multirow{2}{*}{ Vizcaya } & Libres & $-1,178$ & 0,2171 & $-12,039$ & 0,0000 & $\mathrm{I}(1)$ \\
\hline & Protegidas & $-1,592$ & 0,1045 & $-14,766$ & 0,0000 & $\mathrm{I}(1)$ \\
\hline
\end{tabular}

NOTA: * Se ha calculado también el test de Philip Perron para contrastar los resultados del ADF. FUENTE: Elaboración propia a partir de los datos del MFOM.

óptimo ${ }^{12}$ se estima un VAR para ambos procesos con una amplitud temporal entre 2 (Valladolid) y 8 (Madrid y Vizcaya) retardos utilizando únicamente los estadísticamente significativos como resultado de la aplicación de pruebas de máxima verosimilitud $^{13}$.

Las series LIBRES y PROTEGIDAS del modelo de Alicante y Guadalajara se corresponden con una serie integrada y estacionaria, respectivamente. Ello condiciona el

${ }^{12}$ Siendo la razón de verosimilitud (LR), el error de predicción final (FPE), Akaike (AIC), Schwartz (SB) y Hannan-Quinn (HQ) los criterios de información utilizados.

${ }^{13}$ El VAR es estimado después de analizar y corregir estadísticamente el comportamiento de los residuos mediante la introducción de variables dummy, aditiva y/o multiplicativamente, como factores exógenos del sistema. 
análisis al entorno de los modelos autorregresivos a la siguiente expresión del sistema:

$$
\begin{gathered}
\text { LIBRES }_{t}=\alpha_{10}+\sum_{i=1}^{p} \alpha_{1 i} \text { LIBRES }_{t-i} \sum_{i=1}^{p} \beta_{1 i} \text { PROTEGIDAS }_{t-i}+\gamma_{10} Z_{t}+\varepsilon_{1 t} \\
\text { PROTEGIDAS }_{t}=\alpha_{20}+\sum_{i=1}^{p} \alpha_{2 i} \text { LIBRES }_{t-i} \sum_{i=1}^{p} \beta_{2 i} \text { PROTEGIDAS }_{t-i}+\gamma_{20} Z_{t}+\varepsilon_{2 t}
\end{gathered}
$$

donde LIBRES denota un vector de orden $n \times 1$ que recoge la información muestral de la variable endógena vivienda libre en la unidad temporal $t$; PROTEGIDAS, la correspondiente vivienda protegida; $Z$ es una matriz de cambios estructurales; $\alpha y \beta$, coeficientes a estimar correspondientes a valores pasados de las variables endógenas, y $\gamma$ las variables exógenas y $\varepsilon$ el término de perturbación aleatoria distribuido de forma serialmente independiente (Ballabriga, 1991).

El Cuadro 4 recoge los resultados de esta estimación. Contiene los vectores autorregresivos estimados para cada una de las series analizadas con seis $(A C I=31,14273)$ y siete retardos $(A C I=26,21965)$, respectivamente, para Alicante y Guadalajara, y 3 y 4 para Cáceres y Zaragoza, mostrando en todos los casos, una capacidad explicativa sistemáticamente mayor del modelo de libres que de protegidas.

En el caso de Alicante, el modelo de vivienda libre explica el 87,5 por 100 de la variación de la variable dependiente $\left(A d j R_{\text {Alicante }}^{2}=0,87\right)$, mientras que el de vivienda pública es el 67,1 por $100\left(A d j R_{\text {Alicante }}^{2}=0,671\right)$. La ecuación para Alicante aporta evidencia empírica sobre la interrelación positiva entre ambos ciclos. Evidencia la existencia de una relación de causalidad bidireccional en la edificación de Alicante, es decir, la edificación pública afecta positivamente a la libre (con elevado efecto multiplicador de $\beta_{21}=2,24$ ) al igual que al revés, aunque en este caso con un efecto muy débil de reacción inmediata $\left(\beta_{21}=0,047\right)$, mostrando la presencia de efectos crowding in en la edificación, y en consecuencia el carácter endógeno de ambas variables. Esta relación puede justificar el hecho de que no se identifique una relación estadísticamente significativa del shock de 2008 y la extraordinaria caída en ambos ciclos que tiene lugar a partir de entonces ${ }^{14}$. La implicación de este resultado es relevante, reflejando cómo las medidas de incentivo de vivienda pública impulsan la edificación privada sumando sus efectos finales. En este caso,

\footnotetext{
${ }^{14}$ La ecuación para Alicante explica el ciclo de viviendas libres en función de su trayectoria $\left(\alpha_{l i}=0,70\right)$ así como de su vinculación, de igual signo, con la vivienda pública con cinco desfases temporales $\left(\beta_{15}=2,24\right)$, lo que sugiere la existencia de un efecto crowding in en esta provincia. La incorporación de variables de cambio estructural con especificación aditiva $\left(t^{*}=2,86508\right)$ y multiplicativa $\left(t^{*}=-2,60519\right)$ en 2002 contribuye a explicar de forma estadísticamente significativa la evolución del ciclo de viviendas libres. El modelo que relaciona la vivienda pública muestra una pauta de comportamiento similar, con vinculación entre ambos ciclos destacando la significatividad asociada al cambio estructural en 2001 con especificación multiplicativa $\left(t^{*}=3,78797\right)$. Los resultados en esta segunda ecuación ratifican la interrelación positiva entre ambos ciclos y de convergencia (parámetro $\alpha_{25}=-0,034$, negativo y estadísticamente significativo). La aplicación de la prueba de Granger es consistente con lo antes dicho.
} 


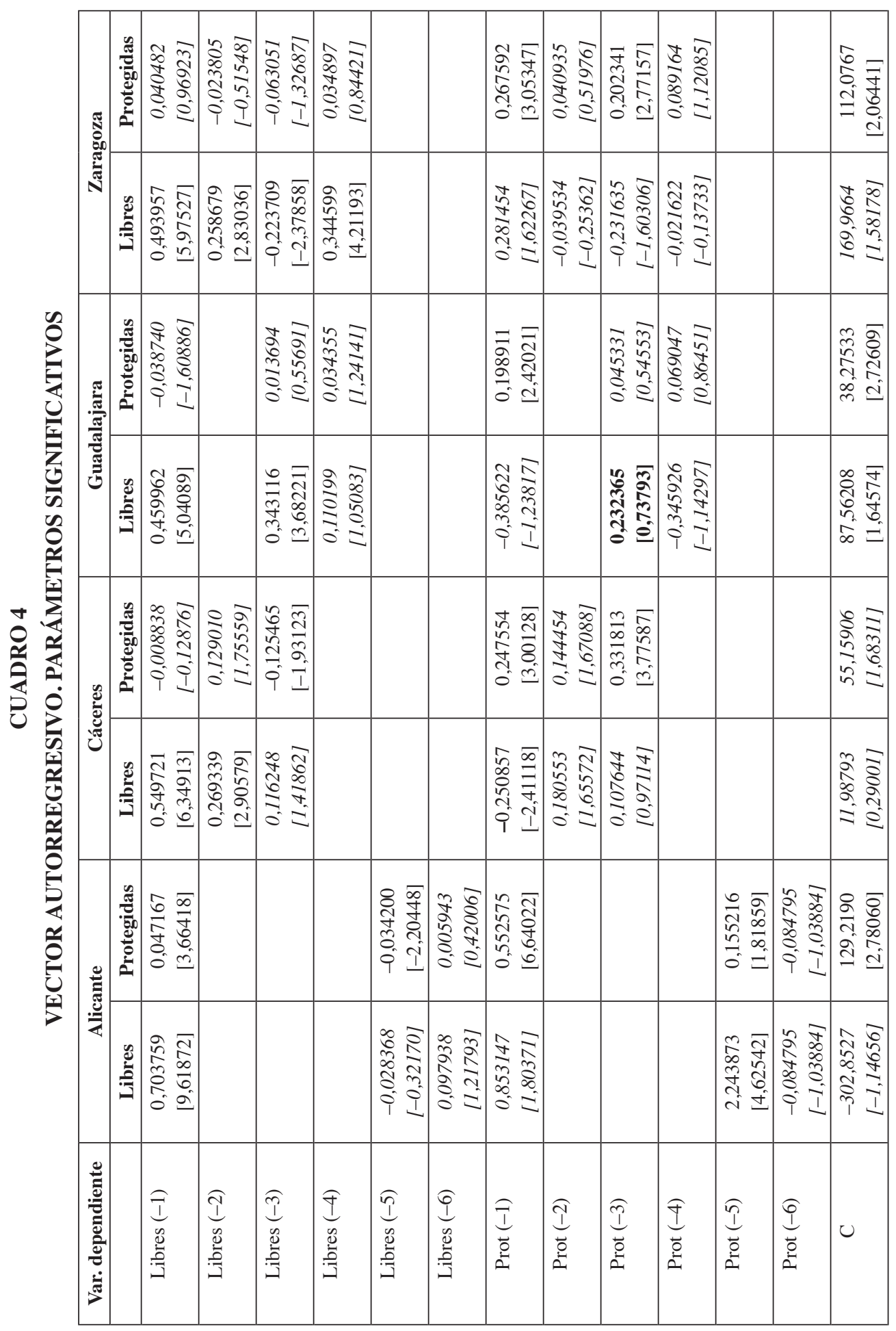




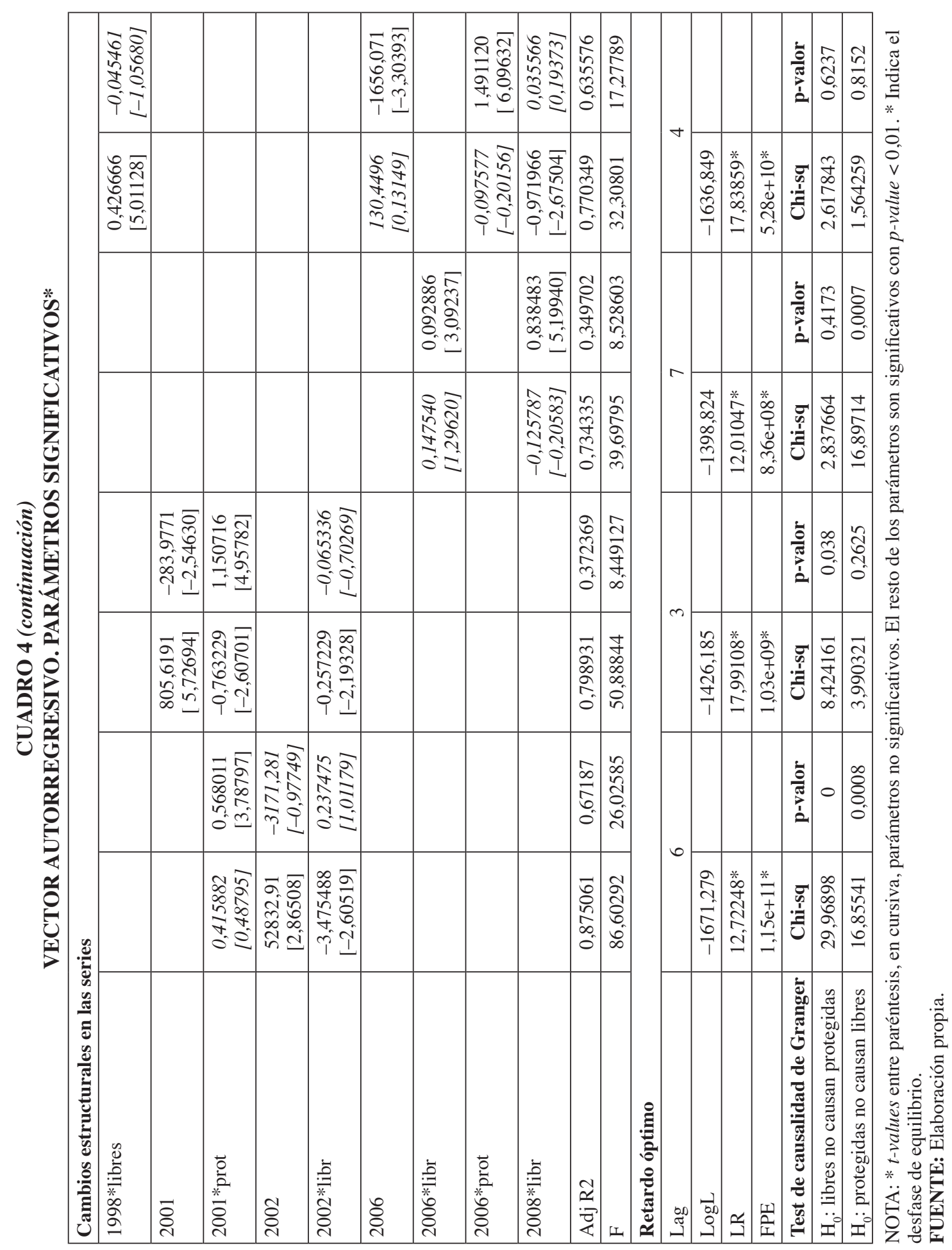


las medidas de política de vivienda tienen un efecto intenso y rápido sobre la producción final.

El modelo de vivienda libre y vivienda pública en Guadalajara se podría explicar sobre la propia base de su dinámica respectivamente sin aparente relación entre los ciclos. En su modelización, destaca el impacto estadísticamente significativo de las variables dummy de cambio estructural, incorporadas con especificación multiplicativa en la modelización del ciclo público en $2006\left(t^{*}=3,09237\right)$ y 2008 $\left(t^{*}=5,19940\right)$. En ambos casos son positivos, pero el parámetro correspondiente al shock de $2008\left(\gamma_{20}=0,838\right)$ indica una fuerte reacción de la vivienda pública coincidiendo con el inicio de los efectos de la Crisis Financiera Global, reflejo de la orientación de la política en ese año. El test de Granger permite visualizar el carácter endógeno de la vivienda pública $(p$-valor $=0,0007)$, pero no biunívoco sino de una sola dirección desde vivienda protegida hacia libre, aunque el modelo en sí no identifica con precisión esta relación. En este caso, los resultados sugieren un efecto individualizado de los ciclos, sin relación entre ellos.

En los casos de Cáceres y Zaragoza, ambas series son no estacionarias. La aplicación del test de Johansen rechaza la hipótesis de existencia de cointegración, por lo que se modeliza también mediante un vector autorregresivo. Para Cáceres ${ }^{15}$, la modelización de la vivienda tanto libre como pública se podría explicar sobre la propia base de su dinámica, aunque el modelo de viviendas libres refleja una relación estadísticamente significativa, y negativa, con las viviendas protegidas (desfasadas un periodo), lo que puede interpretarse como una evidencia empírica de crowding out $\left(\beta_{21}=-0,2508, t^{*}=2,41\right)$. Este efecto no aparece en el sentido contrario, lo que implica una relación de sustitución unidireccional. Destaca también el impacto estadísticamente significativo de los cambios estructurales arriba mencionados asociados a los ciclos privado y público en 2001 y 2002, con un efecto positivo sobre viviendas libres y negativo sobre las privadas en 2001 y un cambio de sentido en 2002 sobre viviendas libres. En este caso, por tanto, el modelo aporta evidencia empírica de que una intervención en la política de vivienda a través del impulso de vivienda protegida tiene un efecto de expulsión sobre la vivienda libre en el periodo analizado y asociado a los shocks temporales.

El modelo de Zaragoza se aproxima mediante un vector autorregresivo con cuatro retardos $(A C I=30,36421)$. La capacidad explicativa de ambos ciclos es estadísticamente significativa y visualiza la dinámica propia de cada serie sin evidencia empírica sobre la existencia de efectos de sustitución, resultados que confirman el test de Granger, mostrando como ambos ciclos son exógenos. De nuevo aquí, las variables

${ }^{15}$ Este modelo se ha estimado con tres retardos $(A C I=26,42540)$ incorporando al mismo como variables exógenas las variables ficticias D2001, D2001*PROTEGIDAS y D2002*LIBRES. Las regresiones que aproximan ambos procesos en esta provincia son estadísticamente significativas con una bondad sensiblemente superior la modelización de la vivienda libre $\left(A d j R^{2}=0,798\right)$ en relación a la vivienda pública $\left(A d j R^{2}=0,3723\right)$. El test de Granger permite visualizar el carácter exógeno de la vivienda pública $(p$-valor $=$ $0,2625)$ y endógeno de la vivienda libre $(p$-valor $=0,0380)$ al 5 por 100 de significación, que es consistente con el efecto contrastado en el ejercicio. 
dummy incorporadas son estadísticamente significativas ${ }^{16}$ en la modelización del ciclo privado en 1998 y 2008, así como en el ciclo público en 2006, todos ellos mostrando un efecto negativo como consecuencia de los cambios estructurales contrastados.

En los casos restantes, las series del conjunto de España, Asturias, Madrid, Valladolid y Vizcaya son I(1) y los test indican que las variables están cointegradas ${ }^{17}$, lo que pone de manifiesto la existencia de un comportamiento sistemático similar en cuanto a la sensibilidad y sentido de reacción durante todo el periodo analizado, que afecta, a su vez, a los propios ciclos. Como es sabido, dos variables integradas de primer orden $I(1)$, están cointegradas cuando existe una combinación lineal de ambas estacionaria de orden cero, $I(0)$, lo que implica la existencia de una relación de equilibrio a largo plazo ${ }^{18}$.

Así pues, en este caso el modelo a estimar para Asturias, Madrid, Valladolid y Vizcaya sería el expresado en [6] y [7].

$$
\begin{gathered}
\Delta(\text { LIBRES })=\alpha_{1}\left(\beta_{10}+\beta_{11} \text { LIBRES }_{1-1}+\beta_{12} \text { PROTEGIDAS }_{t-1}\right)+ \\
+\sum_{i=1}^{p} \alpha_{1 i} \Delta(\text { LIBRES })_{t-i}+\sum_{i=1}^{p} \delta_{1 i} \Delta(\text { PROTEGIDAS })_{t-i}+\gamma Z_{t}+\varepsilon_{1 t} \\
\Delta(\text { PROTEGIDAS })=\alpha_{2}\left(\beta_{20}+\beta_{21} \text { LIBRES }_{1-1}+\beta_{22} \text { PROTEGIDAS }_{t-1}\right)+ \\
+\sum_{i=1}^{p} \alpha_{2 i} \Delta(\text { LIBRES })_{t-i}+\sum_{i=1}^{p} \delta_{2 i} \Delta(\text { PROTEGIDAS })_{t-i}+\gamma Z_{t}+\varepsilon_{2 t}
\end{gathered}
$$

denotando $\triangle($ LIBRES $)$ y $\triangle(P R O T E G I D A S)$ las variables endógenas del modelo, LIBRES y PROTEGIDAS, en diferencias; $\alpha_{i}$, la velocidad de ajuste a corto plazo para ambos ciclos; $\beta$, coeficientes a estimar correspondientes a valores pasados de las variables endógenas en diferencias; $\gamma$ a las variables exógenas, y $\varepsilon$ el término de perturbación aleatoria distribuido de forma serialmente independiente.

Los resultados de la estimación se encuentran en el Cuadro $5^{19}$. A partir de las ecuaciones de cointegración estimadas se evalúa el dominio y significatividad

\footnotetext{
${ }^{16}$ Se contrasta en el caso de la especificación multiplicativa en la modelización del ciclo privado en 1998 $\left(t^{*}=5,01128\right)$ y $2008\left(t^{*}=-2,67504\right)$. Se identifica también un efecto negativo sobre las protegidas de un cambio estructural en $2006\left(t^{*}=-3,303\right)$. El test de Granger permite visualizar la exogeneidad tanto de la vivienda libre ( $p$-valor $=0,6237)$ como de la vivienda pública $(p$-valor $=0,8152)$.

${ }^{17}$ Se ha utilizado el test de Johansen según la prueba de la traza y del máximo valor propio para un nivel de significatividad del uno por ciento para su identificación.

${ }^{18}$ Los sistemas que incorporan en su especificación variables cointegradas, de acuerdo con el teorema de representación de Granger, son normalmente formulados como modelos de corrección de error (MCE) que permiten modelizar las relaciones de largo y corto plazo a la vez, como en $\Delta y_{t}=\gamma\left(\Delta x_{t-i}\right)+\alpha\left(y_{t-1}-\beta_{1}-\beta_{2} x_{t-1}\right)+\varepsilon_{t}$, donde $\alpha\left(y_{t-1}-\beta_{1}-\beta_{2} x_{t-1}\right)$ denota el mecanismo de corrección de error, siendo $\alpha<0 ; \gamma$ mide el efecto a corto plazo que la variable $x_{t}$ ejerce sobre $y_{t} ; \beta_{2}$ recoge el efecto a largo plazo que la variable $x_{t}$ ejerce sobre $y_{t}$, y $\varepsilon_{t}$ el término de perturbación aleatoria. La formulación estacionaria de variables no estacionarias sería, pues, $\Delta y_{t}=\gamma\left(\Delta x_{t-i}\right)+\alpha u_{t-1}+\varepsilon_{t}$, en la que la variación de la variable $y_{t}$ es descrita alrededor de su tendencia a largo plazo en términos del resto de los factores en el modelo, $\Delta x_{t-i}$ integrados de orden cero y la corrección del error $\alpha u_{t-1}=\alpha\left(y_{t-1}-\beta_{1}-\beta_{2} x_{t-1}\right)$, error del equilibrio en el modelo de cointegración (ENGLE y GRANGER, 1987).

19 Todas las regresiones que aproximan ambos procesos son estadísticamente significativas de acuerdo a la prueba $F$-Snedecor presentando una bondad ligeramente superior la modelización del ciclo público en relación al privado.
} 


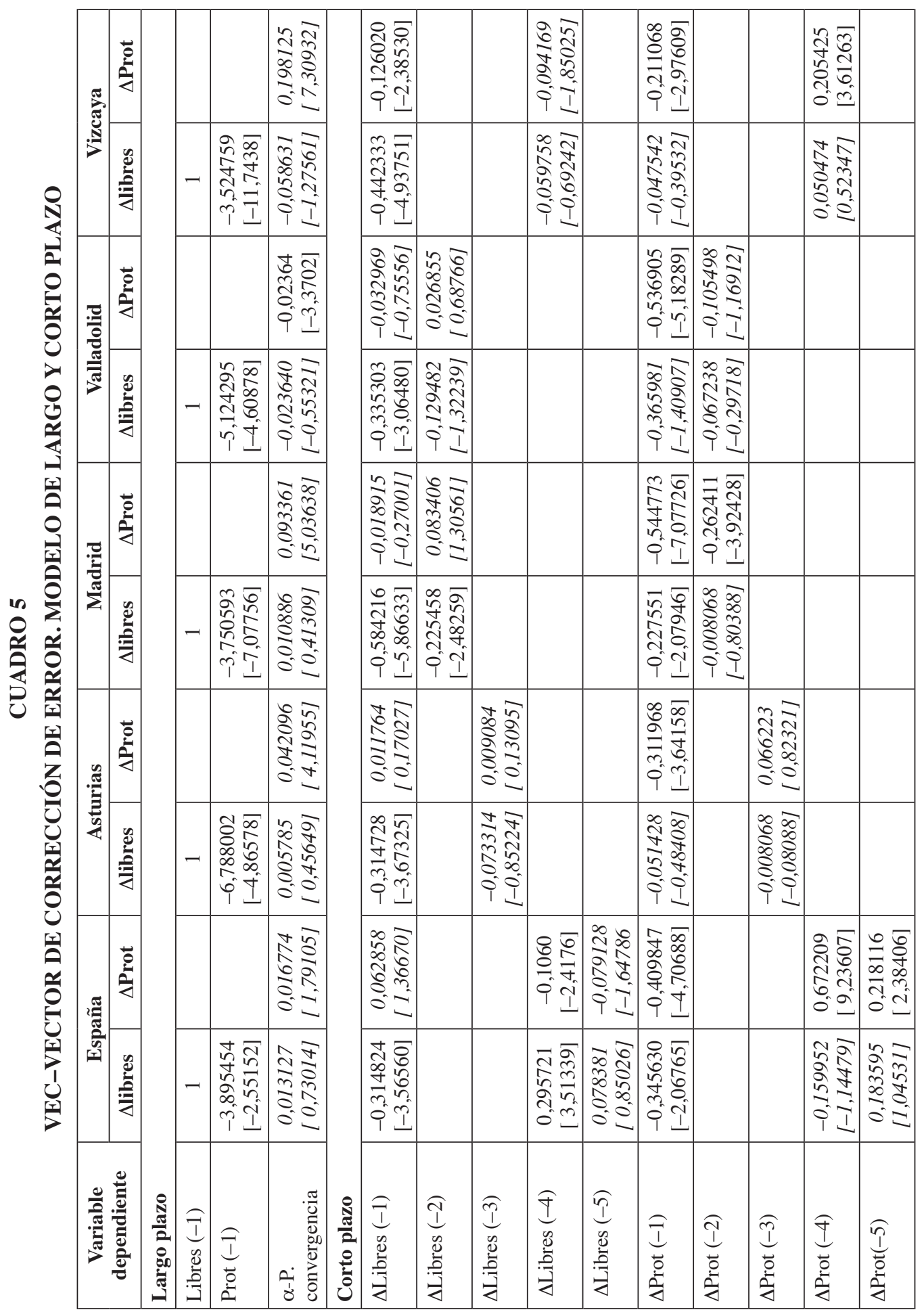




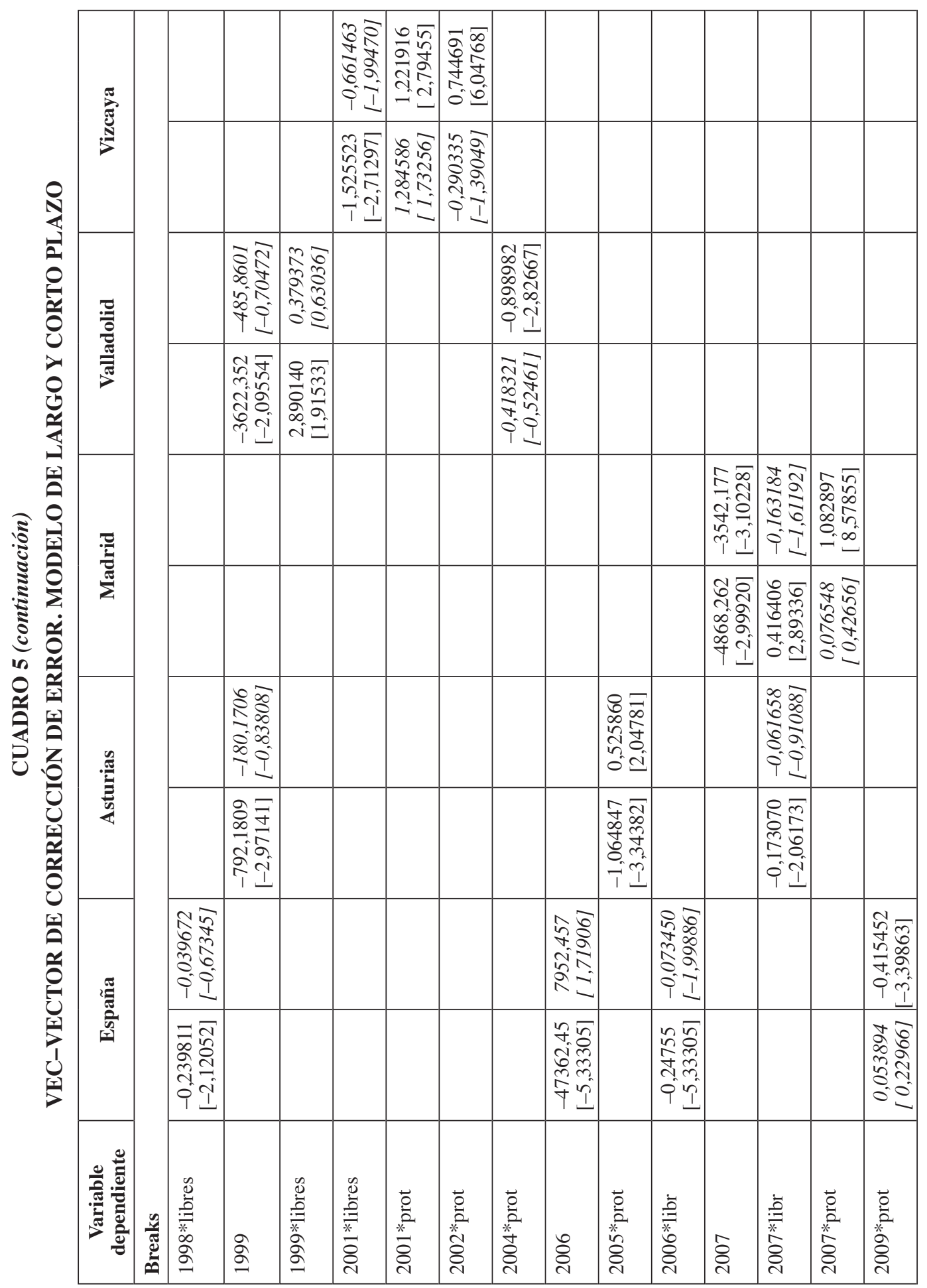




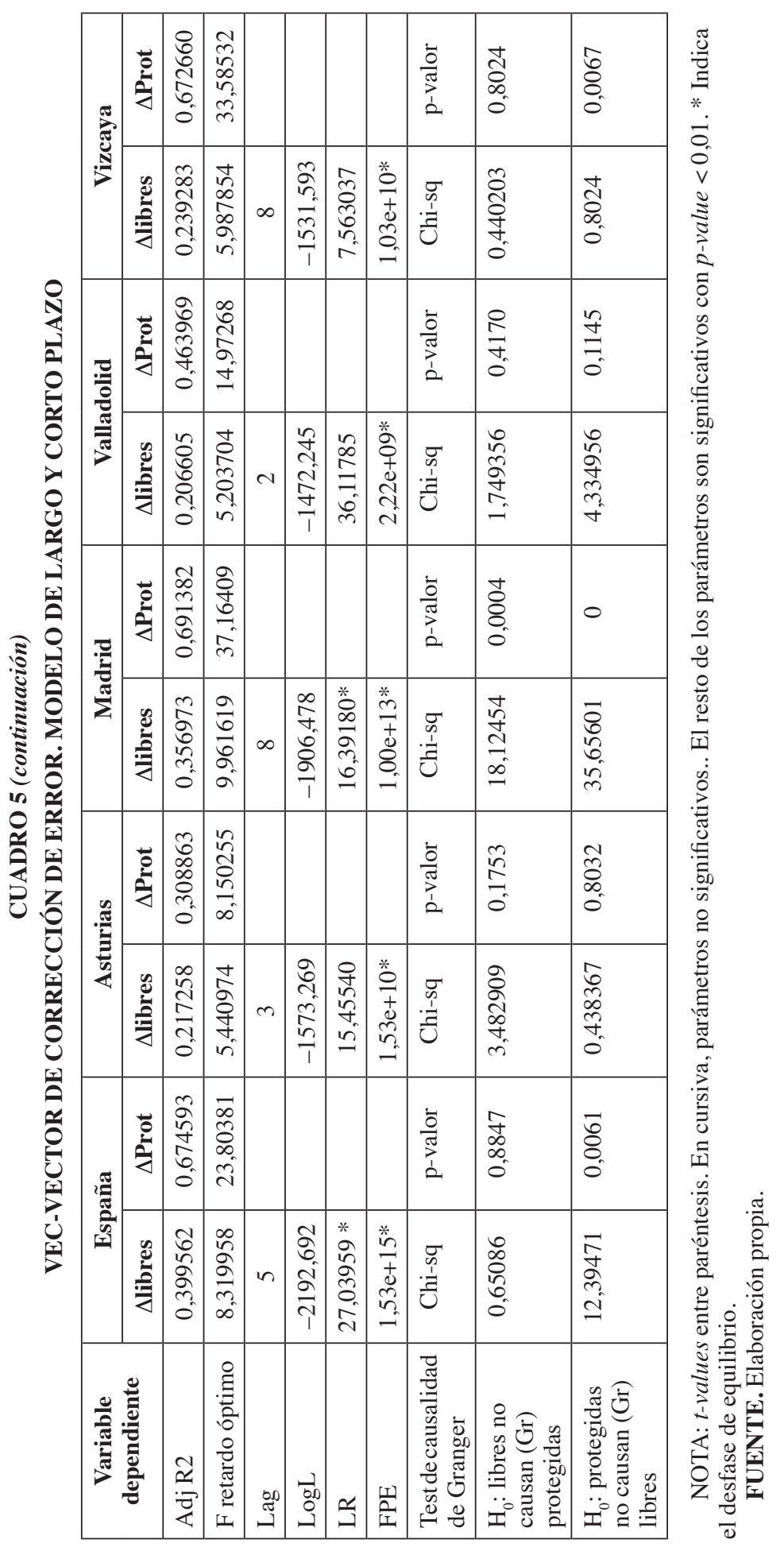


estadística para el ciclo privado en la determinación de la trayectoria del ciclo público del efecto a largo plazo, en relación al corto plazo $^{20}$. Esta relación de largo plazo se interpreta como el reflejo de una situación en la que cada unidad de libres iniciadas se asocia con un número menor de protegidas iniciadas (con el ratio que identifica el parámetro), mostrando un modelo persistente y estable entre ambos ciclos y durante todo el periodo analizado. Este hecho reflejaría la existencia de crowding out sistemático y persistente si su relación es significativa para explicar el crecimiento de las series, en la medida que respaldaría el efecto desplazamiento (causal) sobre las nuevas viviendas iniciadas. El VEC captura esta relación a la par que distingue entre la causalidad del efecto de sustitución procedente de la relación de largo plazo (que tendrá efectos permanentes) y la de corto plazo (que tendrá efectos transitorios). Los efectos transitorios de crowding out que el modelo captura se producen en aquellas provincias donde se obtienen parámetros estadísticamente significativos en la corrección de error (corto plazo).

El modelo estima que estos últimos se dan únicamente a nivel agregado, en España $\left[\delta_{21}=-0,3456\left(t^{*}=-2,0676\right)\right]$ y en Madrid $\left[\delta_{21}=-0,2275\left(t^{*}=-2,0794\right)\right]$ en el sentido del efecto que una acción sobre las protegidas tiene sobre el ciclo de las iniciadas libres. En el sentido contrario (un aumento de las libres desplazando a las protegidas) se ha contrastado en Vizcaya $\left[\alpha_{21}=-0,1260\left(t^{*}=-2,385\right)\right]$.

El término de corrección de error (o parámetro de convergencia) del ciclo privado refleja el efecto sistemático de sustitución en el largo plazo. No es estadísticamente significativo $\triangle$ (LIBRES) en ninguna de las unidades territoriales consideradas, lo que significa que la relación de largo plazo no contribuye a la restauración de la relación de equilibrio de las series cuando se produce una perturbación inesperada en el corto plazo desviándose temporalmente de él. Lo mismo ocurre en la ecuación del ciclo público, $\triangle$ (PROTEGIDAS), salvo para el caso de Valladolid, donde la relación de largo plazo sí contribuye a la consecución del equilibrio en el largo plazo. En este caso, la contribución define una rápida convergencia hacia el equilibrio [CointEq1: $-0,0236\left(t^{*}=-3,3702\right)$ ], que puede interpretarse como una interrelación permanente entre ambos ciclos que corrige su trazado en la dirección de las viviendas públicas. Es decir, las viviendas protegidas iniciadas reaccionan con rapidez restaurando el equilibrio en el mercado de edificación. En este caso, el modelo parece adelantar un efecto de equilibrio a través de la sustitución en el que un aumento en el ciclo público implicaría una reducción inmediata en el privado, lo que es evidencia empírica de crowding out con efectos permanentes.

La evolución en el resto de los casos se explica fundamentalmente a partir de su propia dinámica y de la influencia derivada de las variables dummy que con una especificación aditiva y multiplicativa en referencias temporales localizadas entre 1998 y 2009 justifican cambios estructurales en la dinámica inmobiliaria. Nótese

${ }^{20}$ Se contrasta la significatividad estadística y dominio del primero en los ámbitos analizados, o sea, España $\left[\beta_{22}=-3,8954\left(t^{*}=-2,5515\right)\right]$, Asturias $\left[\beta_{22}=-6,7880\left(t^{*}=-4,8657\right)\right]$, Madrid $\left[\beta_{22}=-3,7505\right.$ $\left.\left(t^{*}=-7,0775\right)\right]$, Valladolid $\left[\beta_{22}=-5,1242\left(t^{*}=-4,6087\right)\right]$ y Vizcaya $\left[\beta_{22}=-3,5247\left(t^{*}=-11,7438\right)\right]$. 
que en los casos de España y de Vizcaya, en la ecuación de viviendas protegidas, el modelo captura un efecto de retroalimentación del ciclo en los desfases 4 y 5 que pueden corresponderse a los ciclos de los planes nuevos de vivienda pública ${ }^{21}$.

De forma adicional al modelo econométrico tradicional, el modelo VAR identifica las interacciones dinámicas que caracterizan el sistema de ecuaciones estimado mediante la construcción de las funciones impulso-respuesta y descomposición de la varianza del error de predicción. Las funciones impulso-respuesta (FIR) recogen la respuesta del sistema a shocks no anticipados en las variables que se capturan en los componentes del vector aleatorio, y son calculadas en este artículo como elemento adicional para contrastar la consistencia de los resultados anteriores y evaluar: 1) cómo se anticipa la reacción de las variables explicadas en el sistema ante el efecto de un $s h o c k^{22}$, específicamente sobre el comportamiento y relaciones de las variables en tiempo, dirección, duración e intensidad, y 2) el grado de endogeneidad de cada variable ${ }^{23}$. Los resultados pueden verse en los Gráficos 4 y 5.

Lo que estos gráficos muestran es la evidencia entre ambos ciclos de edificación tras un shock que se alcanza en las provincias de Alicante, Cáceres, Guadalajara y Zaragoza (menos de 30 desfases), mientras que los impactos tienen efectos permanentes en el resto de los ámbitos, para las viviendas libres.

A modo de síntesis, el efecto crowding out, de expulsión de la construcción de viviendas públicas sobre las privadas, se identifica en este trabajo como un efecto persistente entre los modelos, permitiendo llegar, en algunas regiones, a distinguir entre efectos permanentes y efectos transitorios de crowding out.

La provincia de Alicante es el único caso en que ambos ciclos se encuentran retroalimentados, de manera que ambos se influyen de manera positiva y en las dos direcciones, perfilando un proceso de crowding in circular que está posiblemente relacionado con condiciones de flexibilidad en los procesos edificatorios o en aspectos institucionales.

En el resto de los casos, se encuentra evidencia empírica de la existencia de crowding out en el sentido de cómo los incentivos a la edificación pública afectan de forma negativa a la privada, expulsando parte de ella. Esto ocurre, en las dos direcciones, en el caso de Cáceres; en la dirección de vivienda pública a la privada, en Madrid; y en la dirección contraria (es decir, el aumento en la vivienda libre expulsa a la pública), en Vizcaya.

${ }^{21}$ Dado que el término de corrección de error (CointEq1) del ciclo privado, $\Delta($ LIBRES) no es estadísticamente significativo en ninguna de las unidades territoriales consideradas, se estimó un modelo restringido alternativo en el que suponíamos nulo el parámetro de cointegración de la primera ecuación (Libres). La evidencia empírica sugiere que esta práctica genera estimaciones más estables de las descomposiciones transitorias y permanentes de los shocks (GONZALO y NG, 2001). Los resultados obtenidos han sido muy próximos a los aquí presentados por los que no han sido incluidos en el artículo.

${ }^{22}$ Una alteración en el comportamiento de una variable afectará directamente a la misma y se transmitirá al resto a través de la estructura dinámica que representa el modelo VAR.

${ }^{23} \mathrm{Si}$ una proporción importante de la varianza de una variable se explica por las aportaciones de sus propias perturbaciones, dicha variable sería relativamente más exógena que otras. 


\section{GRÁFICO 4 \\ FUNCIÓN IMPULSO-RESPUESTA}
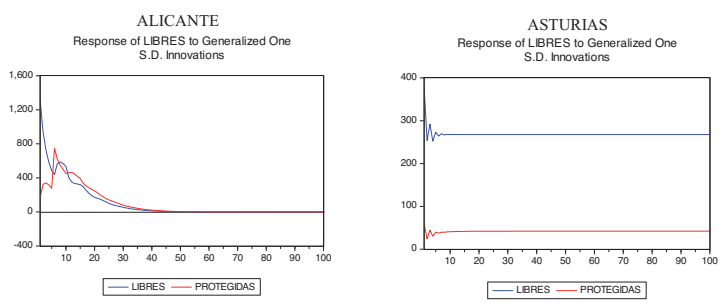

CACERES

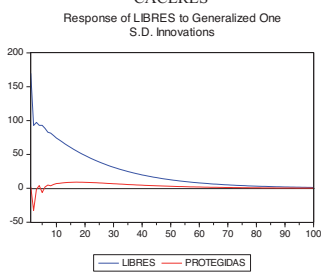

Response of PROTEGIDAS to Generalized One
S. . Innovations

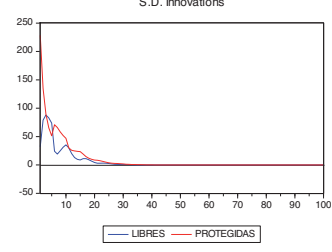

Response of PROTEGIDAS to Generalized One

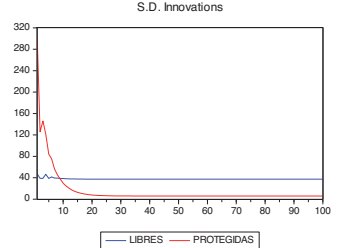

S.D. Innovato to Generalized One

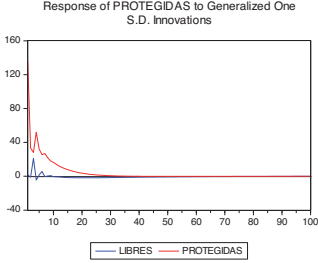

ESPAÑA

Response of LIBRES to Generalized One
S.D. Innovations

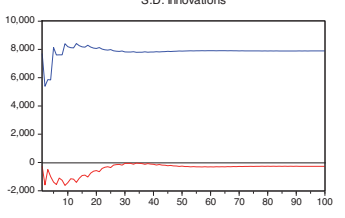

GUADALAJARA

Response of LIBRES to Generalized One
S.D. Innovations

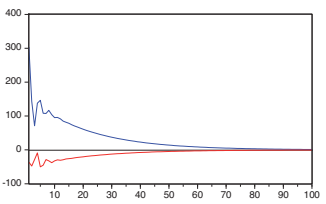

- LIBRES - PROTEGIDAS

Response of PROTEGIDAS to Generalized One

S.D. Inovation
S.

S.D. Innovations
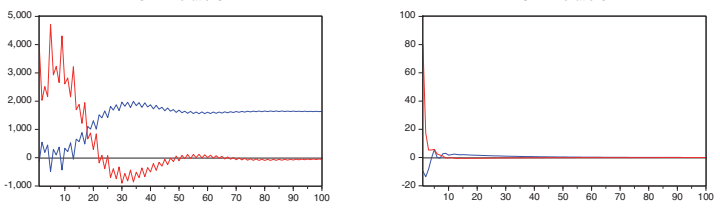

Response of PROTEGIDAS to Generalized

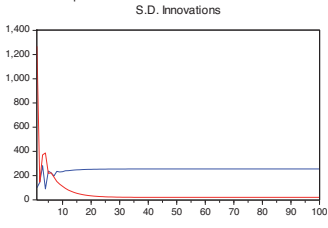

- LERES — PROTEGIDAS

VALLADOLID

—LBBRES —PROTEGIDAS

VIZCAYA

- LBRES — PROTEGIDAS

ZARAGOZA

Response of LBRES to Generalized One
S.D. Innovations

S.D. Innovations

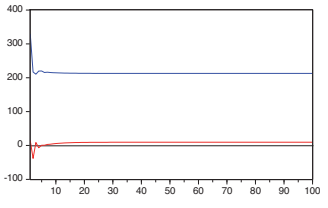

of LIBRES to Gene
S.D. Innovations

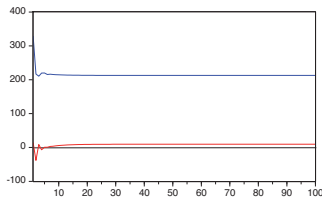

—LBRES — PROTEGIDAS

—LIBRES — PROTEGIDAS

Response of PROTEGIDAS to Generalized One

Response of PROTEGIDAS to Generalized One

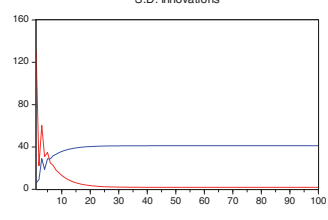

- LIBRES - PROTEGIDAS

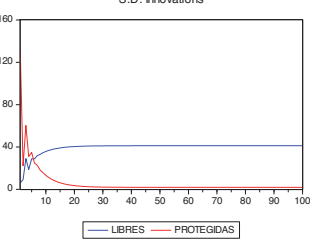

FUENTE: Elaboración propia.

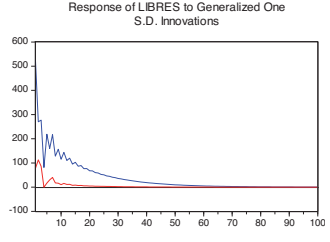

—LIBRES — PROTEGIDAS

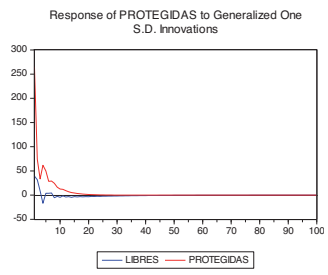


GRÁFICO 5

DESCOMPOSICIÓN DE LA VARIANZA
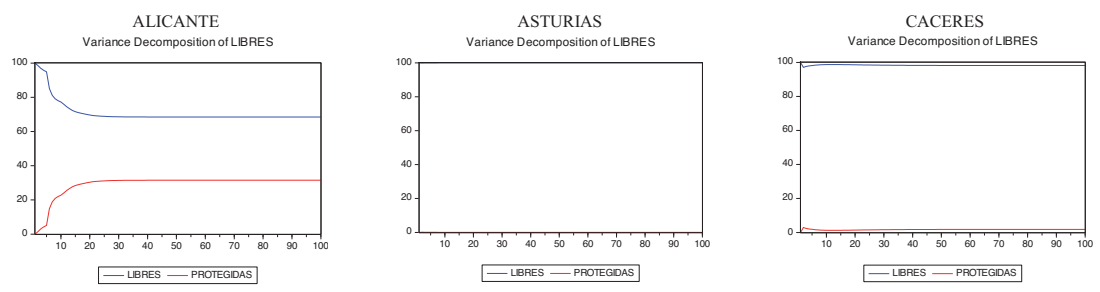

ance Decomposition of PROTEGIDAS

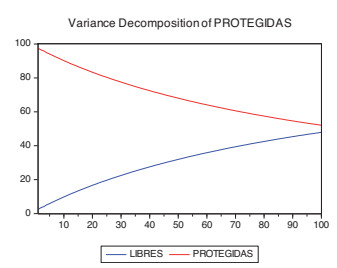

— LIBRES — PROTEGIDAS
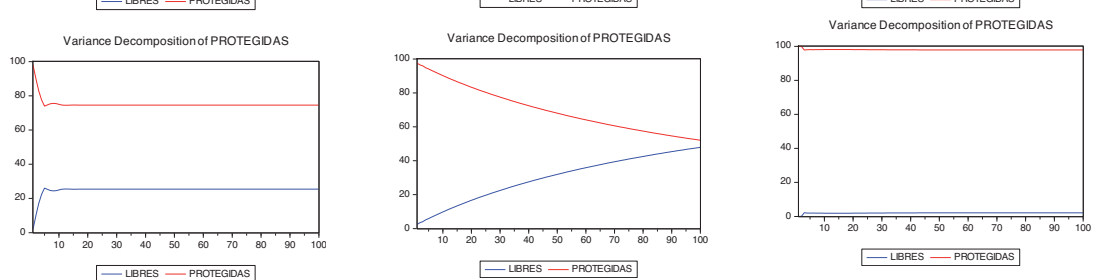

ESPAÑA

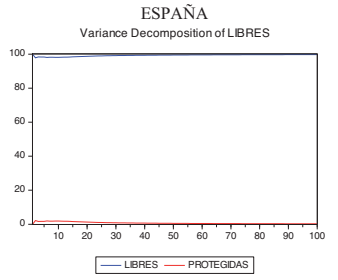

GUADALAJARA
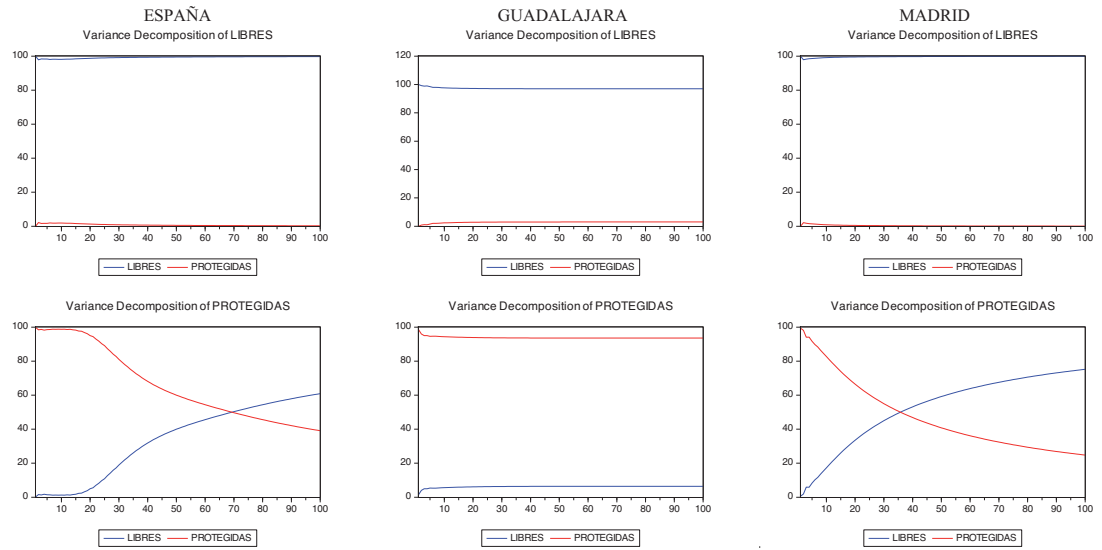

VALLADOLID

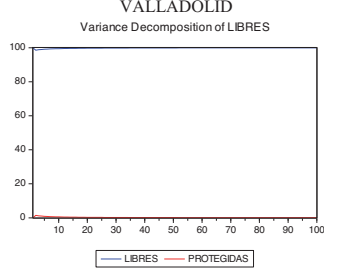

VIZCAYA

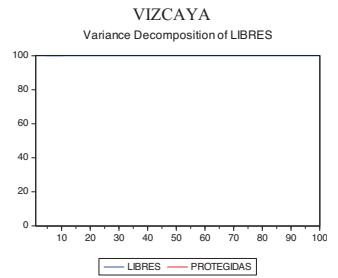

ZARAGOZA

Variance Decomposition of LIBRES

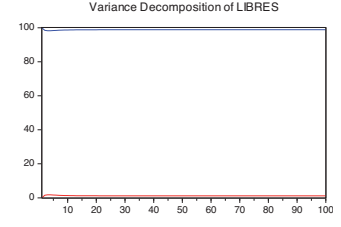

- LIBRES - PROTEGIDAS

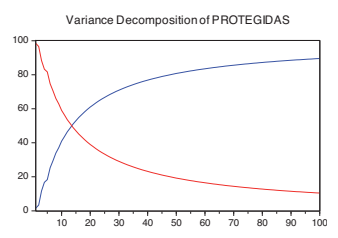

—LIBRES _PROTEGIDAS

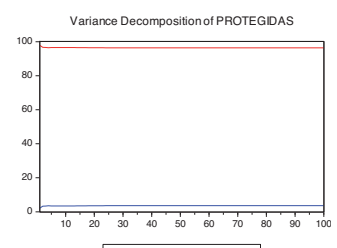

- LBRES — PROTEGIDAS

FUENTE: Elaboración propia. 
No se puede aportar evidencia empírica sobre la existencia de estos efectos de sustitución en las provincias de Guadalajara, Zaragoza y Asturias.

En los casos del conjunto de España, Asturias, Madrid, Valladolid y Vizcaya, los resultados muestran la existencia de un comportamiento sistemático (y negativo) entre ambos ciclos de edificación, lo que implicaría un efecto crowding out persistente en el largo plazo. Sin embargo, este comportamiento no condiciona los cambios de las viviendas libres ni públicas, lo que significa que, en estas provincias, los ciclos se relacionan solo en el corto plazo como se ha resumido arriba. Solo en el caso de Valladolid, el ejercicio aporta evidencia empírica sobre que la relación negativa entre ambos ciclos afecta, de forma sistemática, al ajuste de vivienda pública dibujando el único efecto crowding out de largo plazo identificado, que determina el ajuste de las unidades públicas.

\section{Conclusiones}

El presente artículo desarrolla un análisis para explorar e identificar la existencia de efectos crowding entre las viviendas iniciadas privadas y públicas en España y en 8 provincias, de manera que se pueda establecer si existe el fenómeno de sustitución en la inversión inmobiliaria privada como consecuencia de un aumento en la inversión de viviendas públicas. Las provincias han sido seleccionadas como casos representativos acorde con la evolución de los precios residenciales.

Los efectos para los que se busca evidencia empírica son de expulsión (crowding out) o de empuje (crowding in) de la construcción privada ante acciones de incentivo a la edificación pública, y se identifican a través de las relaciones dinámicas entre los ciclos que permite estimar los métodos de análisis de series temporales. La metodología de análisis utiliza el método de Johansen para el estudio de la trayectoria y dirección de las relaciones entre los ciclos, sobre las series temporales de viviendas iniciadas libres y protegidas para los territorios seleccionados. Tras el análisis de estacionariedad de las series, el ejercicio empírico se desarrolla estimando, por un lado, vectores autorregresivos para aquellas variables que no presentan relación de cointegración (Alicante, Cáceres, Guadalajara y Zaragoza), y modelos de corrección de error para el resto de los casos (España, Asturias, Madrid, Valladolid y Vizcaya). Los resultados se ratifican con un análisis de causalidad mediante el cálculo de las funciones de impulso-respuesta generalizadas.

Los resultados identifican ambos efectos y su intensidad y dirección dispar a lo largo de la geografía española. Se identifican efectos de crowding out en Cáceres (con las viviendas libres siendo endógenas), y también en Madrid así como en el modelo agregado para el conjunto de España. En el primer caso, el efecto es completo mientras que en los dos últimos, los efectos de expulsión se producen solo en el corto plazo, mostrando los efectos transitorios de las intervenciones de política de vivienda nueva. En Valladolid se identifica un efecto de ajuste con efectos permanentes de las viviendas públicas iniciadas al equilibrio en el mercado, 
lo que indicaría un cierto carácter de adaptación de la política pública al status quo del mercado, lo que gana sentido con el impacto de la crisis sobre la edificación, como reacción de política contracíclica. El efecto crowding out, pero en la dirección contraria a la aquí analizada, es decir, el efecto de expulsión de las vivienda públicas como resultado del aumento de las privadas, se detecta en Vizcaya y también para el modelo agregado. Alicante es la única provincia de las estudiadas donde se muestra un efecto de empuje (crowding in) de ambos ciclos remarcable, con un desfase de 5 trimestres, relación bidireccional y con ambos ciclos endógenos.

También se identifican regiones donde no se produce una aparente interrelación y los ciclos privados y públicos reaccionan de forma independiente. Por ejemplo, en Guadalajara y Zaragoza, el ciclo público muestra un claro efecto de trayectoria y refleja la reacción de la política pública de carácter contra cíclico ante el shock de 2008 en la primera provincia, y en 2006 en la segunda. En el caso de Zaragoza, los test muestran que no existe relación causal alguna entre los ciclos mientras que en Guadalajara, el ciclo privado es endógenamente determinado en el modelo.

Estos efectos dispares son el resultado de la aplicación de similares medidas de política de vivienda y su interacción con la dinámica específica de oferta y demanda en cada mercado. Como es conocido, los mercados residenciales son locales y su reacción depende fuertemente de sus características, por lo que es razonable pensar que las medidas de política homogéneas tendrán diferentes resultados en cada uno. Este es un campo que requiere de investigación adicional para que sirva a las autoridades para medir con mayor precisión el alcance de las políticas aplicadas.

\section{Referencias bibliográficas}

[1] AMEMIYA, T. (1980). «Selection of Regressors». International Economic Review, 21 (2), 331-354.

[2] BALL, M. (2011). European Housing Review, RICS.

[3] BALL, M.; MEEN, G. y NYGAARD, C. (2010). «Housing supply price elasticities revisited: Evidence from international, national, local and company data». Journal of Housing Economics, 19, 255-268.

[4] BALLABRIGA, F. (1991). «Instrumentos de la metodología VAR». Documento de Trabajo, Banco de España, Servicio de Estudios 9108.

[5] CHUL-IN LEE (2007). «Does provision of public rental housing crowd out private housing investment? A panel VAR approach. Journal of Housing Economics, 16, 1-20.

[6] ENGLE, R. y GRANGER, S. (1987). «Co-Integration and Error Correction: Representation, Estimation, and Testing». Econometrica, 55 (2), 251-276.

[7] DIPASCUALE, D. y WHEATON, W. (1996). Urban Economics and Real Estate Markets, New Jersey. Prentice-Hall .

[8] FREY, BRUNO S. y JEGEN, R. (2000). «Motivation Crowding Theory: A Survey of Empirical Evidence». CESifo, Working Paper Series No. 245.

[9] GONZALO, J. y NG, S. (2001). «A systematic Framework for Analyzing the Dynamic Effects of Permanent and Transitory Shocks». Journal of Econometric Dynamic and Control, 25 (10) 1527-1546. 
[10] GUISÁN, C. (2002). «Causalidad y cointegración en modelos econométricos: aplicaciones a los países de la OCDE y limitaciones de los test de cointegración». Working Paper Series Economic Development, No. 61, Universidad de Santiago de Compostela.

[11] JOHANSEN, S. (1988). «Statistical analysis of cointegration vectors». Journal of Economics Dynamics and Control , 12 issues, 2-3, 231-254.

[12] JOHANSEN, S. (1992). «Determination of cointegration rank in the presence of a linear trend». Oxford Bulletin of Economics and Statistics, 54 (3), 383-398.

[13] MALPEZZI, S. y MACLENNAN, D. (2001). «The long-run price elasticity of supply of new residential construction in the United States and the United Kingdom». Journal of Housing Economics, 10, 278-306.

[14] MALPEZZI, S. y VANDELL, K. (2002). «Does the low-income housing tax credit increase the supply of housing?», Journal of Housing Economics, 11, 360-380.

[15] MUELLBAUER, J.(2007). «Housing, Credit and consumer Expenditure».ProceedingsEconomic Policy Symposium-Jackson Hole, Federal Reserve Bank of Kansas City, 267334.

[16] MURRAY, M. (1983). «Subsidized and unsubsidized housing starts: 1961-1977». The Review of Economics and Statistics, 65 (4), 590-597.

[17] MURRAY, M. (1999). «Subsidized and unsubsidized housing stocks 1935 to 1987: crowding out and cointegration». Journal of Real Estate Finance and Economics, 18, 107-124.

[18] NORDVIK, V. (2006). «Selective housing policy in local housing markets and the supply of housing». Journal of Housing Economics, 15, 279-292.

[19] ORTALO-MAGNÉ, F. y RADY, S. (2006). «Housing market dynamics: On the contribution of income shocks and credit constraints». The Review of Economic Studies, 73 (2), 459-485.

[20] QUIGLEY, J. M.; RAPHAEL, S. y ROSENTHAL, L. (2008). «Measuring Land Use Regulations and their effects in the Housing Market». Berkeley Program on Housing and Urban Policy Working Paper W008-004.

[21] QUIGLEY, J. M. (1997). The Economics of Housing. Edward Elgar Publishing, Massachusetts, vols. 1 y 2.

[22] SIMS, C. (1980). «Macroeconomics and reality». Econometrica, 48, 165-192.

[23] SINAI, T. y WALDFOGEL, J. (2005). «Do low-income housing subsidies increase the occupied housing stock?». Journal of Public Economics, 89, 2137-2164.

[24] TALTAVULL, P. (2003). «La política de vivienda», en J. Salinas J. y S. Álvarez (coords.), El gasto público en la democracia, Instituto de Estudios Fiscales.

[25] TALTAVULL DE LA PAZ, P. (2012). «The Responsiveness of New Supply to House Prices: A Perspective from the Spanish Housing Market», en C. M. Jones, M. White y N. Dunse, Challenges of the Housing Economy: An International Perspective. WileyBlackwell, Londresn.

[26] TALTAVULL DE LA PAZ, P. (2014). «New housing supply and price reactions: Evidence from Spanish markets». Journal of European Real Estate Research, 7 (1), 4-28.

[27] TALTAVULL DE LA PAZ, P. y WHITE, M. (2012). «Fundamental Drivers of House Price Change: The Role of Money, Mortgages, and Migration in Spain and the United Kingdom». Journal of Property Research, 29 (4), 341-367.

[28] WHITEHEAD, C. (2003). «The economics of social housing», en T. O’Sullivan y K. Gibb (eds.), Housing Economics and Public Policy. Londres, Blackwell Science. 
